\begin{tabular}{|c|c|}
\hline Title & Oxidative Generation of 1-Nitroalkyl Radicals and Their A ddition Reaction to Olefins \\
\hline Author(s) & A rai, Noriyoshi; Narasaka, Koichi \\
\hline Citation & $\begin{array}{l}\text { Bulletin of the Chemical Society of Japan, } 70(10), 2525-2534 \\
\text { https://doi.org/10.1246/pcsj. } 70.2525\end{array}$ \\
\hline Issue Date & 1997 \\
\hline Doc URL & http:/hdl.handle.net/2115/0769 \\
\hline Type & article \\
\hline File Information & bcsj .70.2525.pdf \\
\hline
\end{tabular}

Instructions for use 


\title{
Oxidative Generation of 1-Nitroalkyl Radicals and Their Addition Reaction to Olefins ${ }^{\#}$
}

\author{
Noriyoshi Arai and Koichi Narasaka* \\ Department of Chemistry, Graduate School of Science, The University of Tokyo, 7-3-1, Hongo, Bunkyo-ku, Tokyo 113
}

(Received April 24, 1997)

\begin{abstract}
1-Nitroalkyl radicals are generated by oxidation of potassium salt of 1-aci-nitroalkanes with ammonium hexanitratocerate(IV). When the oxidation is carried out in the presence of electron-rich olefins, such as silyl enol ethers, intermolecular addition of the radicals onto the olefins proceeds to afford $\beta$-nitro ketones, which are further converted to $\alpha, \beta$-unsaturated ketones in high yield. Stereoselective construction of fused ring systems is achieved by intramolecular addition of 1-nitroalkyl radicals.
\end{abstract}

Carbon-carbon bond-forming reactions by using radical species are recently attracting much attention in synthetic chemistry because of their unique reactivity, distinct from that of ionic species. ${ }^{1,2}$ Though one-electron oxidation of carbanions is considered to be one of the simplest methods for generation of carbon-centered radicals, this method has rarely been employed as a synthetic tool. That is, although several reactions are known to form carbon-carbon bonds by the use of radicals generated by one-electron oxidation of carbanions, most of them afford self-coupling products and few examples have been reported concerning the intermolecular carbon-carbon bond forming reactions. ${ }^{3-8)}$ In these intermolecular reactions, the yield of the products is not sufficiently high, even with the use of large excess amounts of radical acceptors. Recently, we found that 1nitroalkyl radicals are generated by oxidation of aci-nitro anions with ammonium hexanitratocerate(IV) (CAN), and the intermolecular addition reaction proceeds with electronrich olefins. ${ }^{9)}$ In this paper, we would like to report the details of this reaction and the application to the construction of fused ring systems by intramolecular addition reaction of thus generated 1-nitroalkyl radicals.

\section{Results and Discussion}

Intermolecular Addition Reaction between 1-Nitroalkyl Radicals and Electron-Rich Olefins. Though it has been known that one-electron oxidation of aci-nitro anions with a metallic oxidant generates 1-nitroalkyl radicals, their addition reaction to olefins has rarely been reported. ${ }^{10-14)} \mathrm{Re}$ cently, Bowmann et al. reported an intramolecular cyclization of 1-nitroalkyl radical using a norbornene derivative, but, the yield of the cyclized product was not satisfactory. ${ }^{15)}$ In the course of our study on generation of reactive species by oxidation with high valent metallic compounds, we found

\# Dedicated to Professor Dieter Seebach on the occasion of his 60th birthday. an intermolecular addition reaction of 1-nitroalkyl radical to electron-rich olefins. When a solution of potassium salt of 1-nitro-4-phenylbutane (1a) in methanol, prepared by treating 1a with potassium hydroxide, was added to a solution of CAN and (1-trimethylsiloxyethenyl)benzene (2) in methanol at $-78{ }^{\circ} \mathrm{C}(\mathbf{1 a}: \mathrm{KOH}: \mathrm{CAN}: \mathbf{2}=1: 1.3: 1.9: 0.85 ; 0.1$ mol dm ${ }^{-3}$ for 1a), the reaction proceeded to give 3-nitro-1,6diphenyl-1-hexanone (3) as a crude product. Since purification of the $\beta$-nitro ketone 3 by preparative TLC (silica gel) caused a partial elimination of nitrous acid from $\mathbf{3}$, the crude product was purified after converting to 1,6-diphenyl2-hexen-1-one (4a). Thus, by treatment of the crude nitro ketone 3 with triethylamine, the $\alpha, \beta$-unsaturated ketone $4 \mathbf{a}$ was obtained in $35 \%$ yield based on the silyl enol ether $\mathbf{2}$, accompanied by the formation of a self-coupling product 5 (44\%) (Eq. 1).

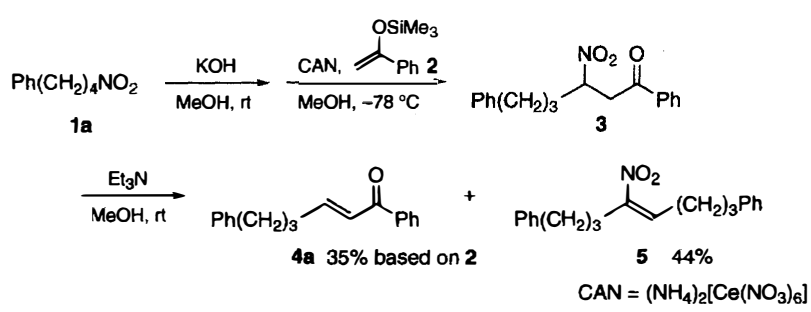

(1)

This reaction is considered to proceed as follows (Scheme 1). A potassium salt of 1-aci-nitroalkane $\mathbf{A}$ is oxidized with CAN to generate a 1-nitroalkyl radical $\mathbf{B}$. The radical species $\mathbf{B}$ adds to the enol ether $\mathbf{2}$, affording a radical intermediate $\mathbf{C}$. Oxidation of the intermediate $\mathbf{C}$ with $\mathbf{C A N}$ gives a cation $\mathbf{D}$, desilylation of which affords the $\beta$-nitro ketone 3. It is well known that, in the alkylation of aci-nitro anions with alkyl halides, generally $O$-alkylation proceeds predominantly over $C$-alkylation. ${ }^{16-18)}$ In the present radical addition to the silyl enol ether, $C$-alkylation proceeded exclusively.

In order to optimize the reaction conditions, effects of the 


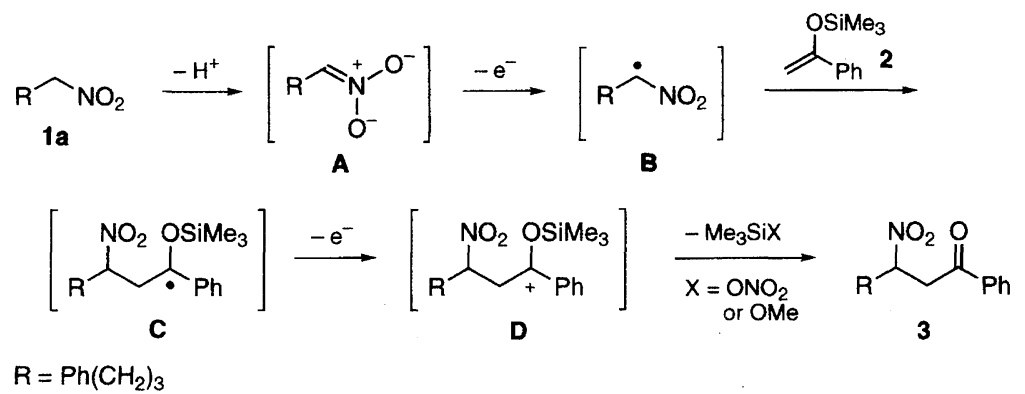

Scheme 1. Mechanism for the addition reaction of the nitroalkane to the silyl enol ether.

Table 1. Temperature and Concentration Effect on the Yield of $\mathbf{4} \mathbf{a}^{\mathrm{a})}$

\begin{tabular}{|c|c|c|c|c|c|}
\hline \multirow[b]{2}{*}{$\underset{\mathbf{P h}\left(\mathrm{CH}_{2}\right)_{3}}{\widehat{\mathrm{N}}}$} & $\mathrm{KOH}$ & ${ }_{\mathrm{CAN}} \stackrel{\mathrm{OSiMe}_{3}}{\lambda_{\mathrm{Ph}}^{2}}$ & $\mathrm{Et}_{3} \mathrm{~N}$ & & \\
\hline & $\mathrm{MeOH}, \mathrm{rt}$ & $\begin{array}{l}\mathrm{MeOH} \\
15 \mathrm{~min}\end{array}$ & $\mathrm{MeOH}$ & & \\
\hline \multirow{2}{*}{ Entry } & Temp & Concn & \multicolumn{3}{|c|}{ Yield/\% } \\
\hline & ${ }^{\circ} \mathrm{C}$ & $\mathrm{mol} \mathrm{dm}^{-3}$ & $4 a^{b)}$ & 5 & $6^{\text {b) }}$ \\
\hline $1^{\text {c) }}$ & -78 & 0.1 & 35 & 44 & 11 \\
\hline $2^{\mathrm{d})}$ & -78 & 0.05 & 74 & 13 & 2 \\
\hline 3 & -78 & 0.03 & 99 & 15 & 0 \\
\hline 4 & -61 & 0.03 & 85 & 5 & 20 \\
\hline 5 & -45 & 0.03 & 55 & 15 & 37 \\
\hline
\end{tabular}

a) $1 \mathrm{a}: \mathrm{KOH}: \mathrm{CAN}: 2=1.0: 1.3: 1.9: 0.85$. b) Based on the silyl enol ether 2. c) A small amount (3\% based on 1a) of 3,4-dihydro-1 $(2 H)$-naphthalenone was obtained. d) A small amount ( $8 \%$ based on 1a) of 3,4-dihydro-1 $(2 H)$-naphthalenone was obtained.

concentration and the reaction temperature were examined first. As shown in Table 1, when the concentration became lower, formation of the self-coupling product $\mathbf{5}$ was diminished and the product was obtained in higher yield (Entries 1,2 , and 3). The reaction temperature is crucial for the selective preparation of $\mathbf{4 a}$. At higher temperature, the yield of the dimer $\mathbf{6}$ of the enol ether was increased considerably (Entries 3,4 , and 5). the enone $\mathbf{4 a}$ was obtained quantitatively by the reaction at $-78{ }^{\circ} \mathrm{C}$ (Entry 3$)$.

The reaction was investigated with some oxidizing agents such as $\left[\mathrm{Fe}\left(\eta^{5}-\mathrm{C}_{5} \mathrm{H}_{5}\right)_{2}\right] \mathrm{PF}_{6}, \mathrm{Fe}\left(\mathrm{NO}_{3}\right)_{3} \cdot 9 \mathrm{H}_{2} \mathrm{O}, \mathrm{Mn}(\text { pic })_{3}$, or
$\mathrm{Ag}(\text { pic })_{2}$ (pic $=2$-pyridinecarboxylato). The addition product $4 \mathbf{a}$ was obtained in $12 \%$ yield only when $\left[\mathrm{Fe}\left(\eta^{5}-\mathrm{C}_{5} \mathrm{H}_{5}\right)_{2}\right]$ $\mathrm{PF}_{6}$ was employed. The use of other oxidizing agents gave only the self-coupling product $\mathbf{5}$, or brought about oxidation or hydrolysis of the silyl enol ether 2 .

In addition to potassium hydroxide, sodium or lithium hydroxides and potassium methoxide can be used as a base to generate the aci-nitro anion, giving $\mathbf{4 a}$ in more than $93 \%$ yield. No reaction took place, however, when a weak base such as triethylamine was used.

Under the optimum reaction conditions found as above, the nitroalkane 1a reacted with various silyl enol ethers to afford the corresponding addition products in good yield (Table 2, Entries 2, 3, and 4). The reactions with a tetrasubstituted silyl enol ether (Entry 5) and cyclic silyl enol ethers (Entries 6 and 7), however, gave poor yields of the products. In addition to silyl enol ethers, a vinyl ether, a styrene derivative, and an allylsilane could be employed successfully to give the corresponding products in moderate to good yield (Entries 8, 9 , and 10). The formation of the unexpected hydroxy ketone 15a is explained as follows (Scheme 2): A cation intermediate $\mathbf{E}$ is formed by the addition of the 1-nitroalkyl radical to $\alpha$-methylstyrene and successive oxidation. Trapping of the cation species $\mathbf{E}$ by the solvent $(\mathrm{MeOH})$ affords the product $\mathbf{1 4 a}$, while intramolecular capture of $\mathbf{E}$ by the nitro group gives a cyclic compound $\mathbf{F}$. Hydrolysis of $\mathbf{F}$ in $\mathrm{S}_{\mathrm{N}} 1$ manner under acidic work-up conditions would easily take place to generate aci-nitro compound $\mathbf{G}$, which is hydrolyzed to the ketone $\mathbf{1 5 a}$ by Nef-type reaction.

The reactions of various nitroalkanes and silyl enol ethers were examined as shown in Table 3 . When primary nitroalkanes such as 5-nitropentyl acetate (1b) and a silyl ether of

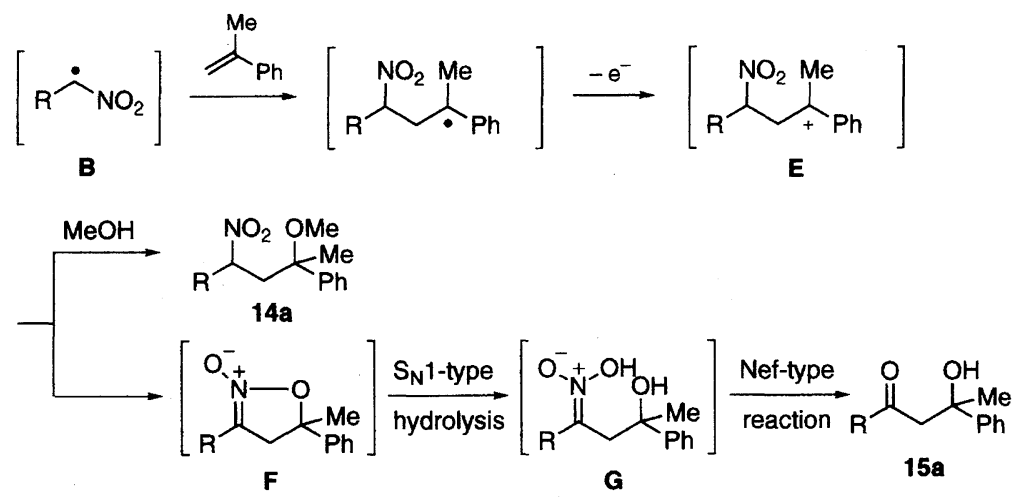

Scheme 2. Formation of the hydroxy ketone $\mathbf{1 5 a}$. 
Table 2. Reaction of 1-Nitro-4-phenylbutane (1a) with Various Olefins ${ }^{\text {a) }}$

$$
\begin{aligned}
& \mathrm{R}_{\mathrm{NO}_{2}} \underset{\mathrm{MeOH}, \mathrm{rt}}{\stackrel{\mathrm{KOH}}{\mathrm{MeOH},-78^{\circ} \mathrm{C}}} \frac{\mathrm{CAN} \text {, Olefin }}{\mathrm{MeOH}, \mathrm{rt}} \text { Product } \\
& 1 a \quad 15 \mathrm{~min} \\
& \mathrm{R}=\mathrm{Ph}\left(\mathrm{CH}_{2}\right)_{3}
\end{aligned}
$$

\begin{tabular}{|c|c|c|c|c|}
\hline Entry & Olefin & Product & & Yield/\% $\%^{\text {b) }}$ \\
\hline 1 & & & & 99 \\
\hline 2 & & & & 81 \\
\hline 3 & & & $8 a$ & 78 \\
\hline 4 & & & $9 a$ & 79 \\
\hline 5 & & & $10 a$ & 5 \\
\hline 6 & & & 11 & 28 \\
\hline 7 & & & 12) & 38 \\
\hline $8^{\text {d) }}$ & & & $13 a$ & 96 \\
\hline $9^{\text {d) }}$ & & & & $48^{\mathrm{e})} 25^{\mathrm{e})}$ \\
\hline $10^{\mathrm{d})}$ & SiMe & & $16 a$ & 45 \\
\hline
\end{tabular}

a) 1a: $\mathrm{KOH}: \mathrm{CAN}:$ olefin $=1.0: 1.3: 1.9: 0.85$. b) Based on the olefin. c) $Z / E=95 / 5$

(determined by ${ }^{1} \mathrm{HNMR}$ ). d) The crude products were not treated with triethylamine.

e) The yield was based on the nitroalkane 1a, since 4 molar amounts of olefin were used.

3-nitro-1-propanol 1c were employed, the addition products were obtained in moderate yield (Entries 2, 3, 4, and 5). A secondary nitroalkane 1d and a cyclic nitroalkane 1e also afforded $\alpha, \beta$-unsaturated ketones in excellent yield in the reaction with 2 (Entries 6 and 8), while the reactions with an aliphatic silyl enol ether resulted in poor yield of the products (Entries 7 and 9). Phenylnitromethane (1f) gave a complex mixture (Entry 10). Although the oxidation of nitromethane (1g) afforded the corresponding $\beta$-nitro ketone, the isolated yield was hard to determine because of the instability of the $\beta$-nitro ketone and the volatility of acrylophenone (4g) which was formed by the elimination of nitrous acid from the $\beta$-nitro ketone. Only NMR yield of $\mathbf{4 g}$ was shown in the table (Entry 11) ${ }^{19)}$ When the initially formed $\beta$-nitro ketones resist the elimination to $\alpha, \beta$-unsaturated ketones, the use of DBU is preferable. In these cases, methanol should not be employed as solvent, since its Michael addition proceeds on produced $\alpha, \beta$-unsaturated ketones.

Construction of Fused Ring Systems by the Intramolecular Cyclization of 1-Nitroalkyl Radicals. As described above, the oxidation of aci-nitro anions with $\mathrm{CAN}$ in the presence of an electron-rich olefin affords the correspond- ing addition products. This carbon-carbon bond-forming reaction was applied for constructing fused ring systems by intramolecular cyclization. When nitro compounds $17 \mathbf{a}$ and 17b were treated with potassium hydroxide, followed by oxidation with CAN, the cyclized products $18 \mathrm{a}$ and $\mathbf{1 8 b}$ were obtained, respectively, as a single stereoisomer (Eq. 2). The relative configuration of $\mathbf{1 8 b}$ was unambiguously determined as trans by $\mathrm{X}$-ray crystallographic analysis. ${ }^{20)}$ Introduction of substituents on the olefinic moiety is essential for stabilizing intermediate radical species. In fact, a terminal vinyl compound 17c did not give desired cyclized products cleanly but a mixture which was difficult to separate.

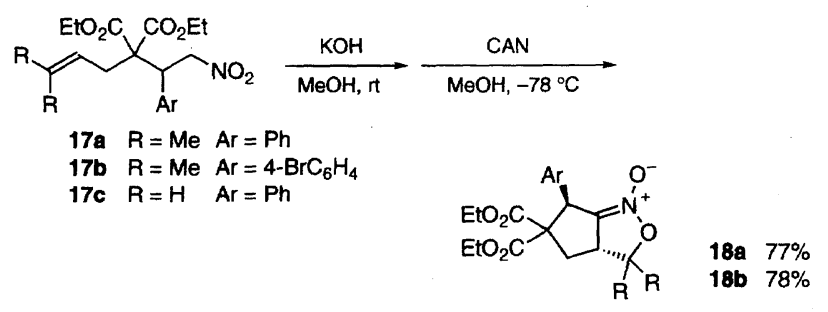

This cyclization was further applied to six-membered ring 
Table 3. Reaction of Various Nitroalkanes with Silyl Enol Ethers ${ }^{\text {a) }}$

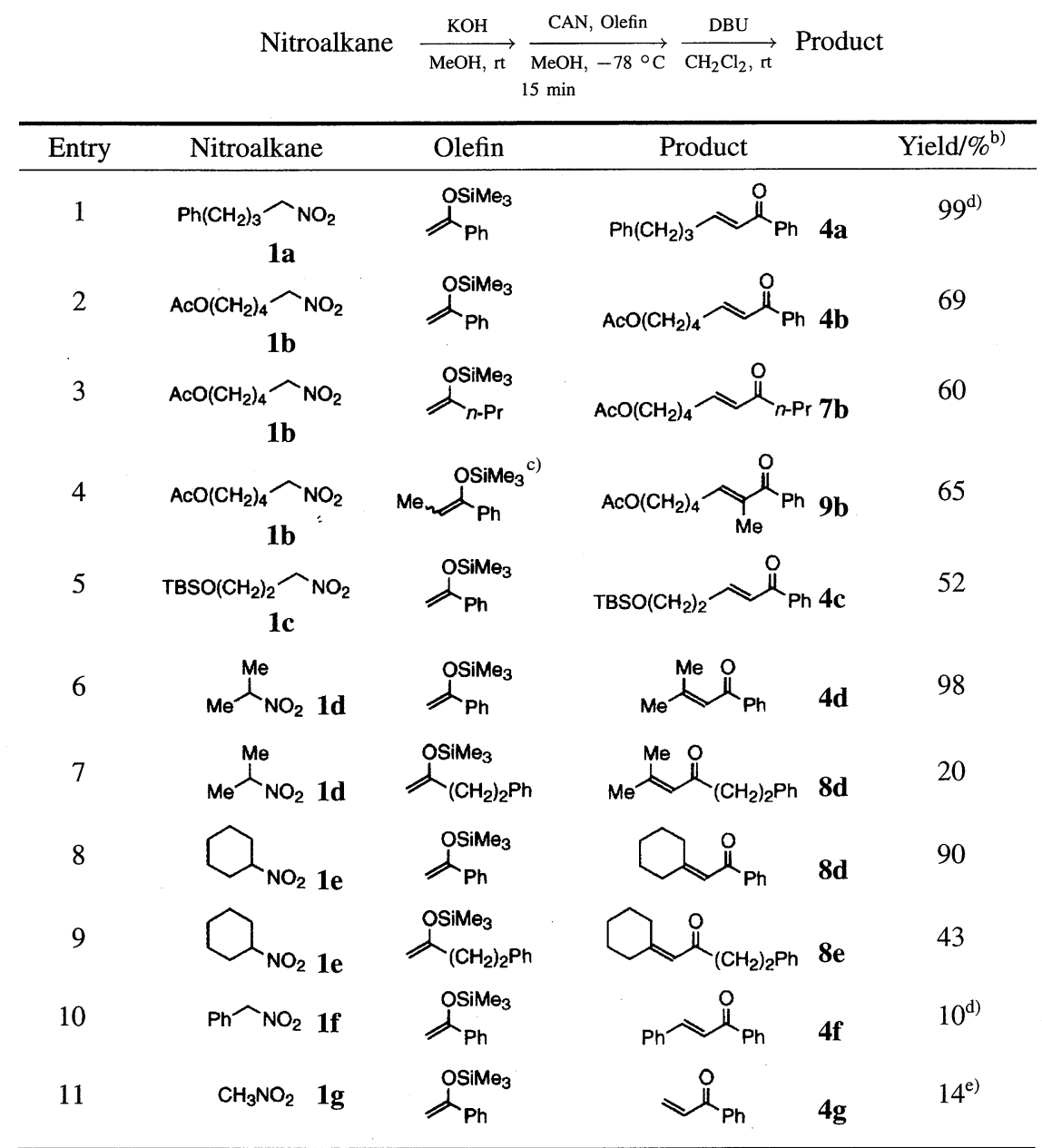

a) nitroalkane : $\mathrm{KOH}: \mathrm{CAN}:$ olefin $=1.0: 1.3: 1.9: 0.85$. b) Based on the olefin.

c) $Z / E=95 / 5$ (determined by ${ }^{1} \mathrm{HNMR}$ ). d) The crude products were treated with triethylamine instead of DBU. e) Determined by ${ }^{1} \mathrm{HNMR}$ using $\mathrm{Ph}_{3} \mathrm{CH}$ as an internal standard.

formation. For example, potassium salt of a nitro compound $\mathbf{1 9}$ cyclized to cyclohexanes $\mathbf{2 0}$ and 21 in good total yield (Eq. 3).
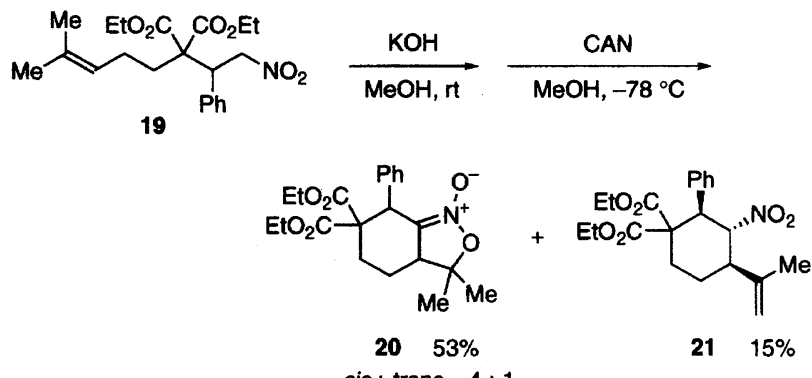

(3)

The product $\mathbf{2 0}$ was obtained as a mixture of two stereoisomers (cis: trans $=4: 1$ ). The isomer ratio was determined by the integration value of ${ }^{1} \mathrm{HNMR}$. Relative configuration was determined by comparing the coupling-constant between $\mathrm{H}_{\mathrm{a}}$ and $\mathrm{H}_{\mathrm{b}}$ with that of $\mathbf{1 8 a}$, whose relative configuration was

determined as mentioned above (Fig. 1). It was confirmed by molecular mechanics calculation that the location of $\mathrm{H}_{\mathrm{a}}$ and $\mathrm{H}_{\mathrm{b}}$ in the most stable conformer of the trans-20 is similar to that of $\mathrm{H}_{\mathrm{c}}$ and $\mathrm{H}_{\mathrm{d}}$ in $\mathbf{1 8 a}$, so that each coupling-constant is expected to have nearly the same value. ${ }^{21)}$

Though we tried a seven-membered ring formation from 22, the desired cyclized product $\mathbf{2 3}$ was obtained only in low yield (Eq. 4).

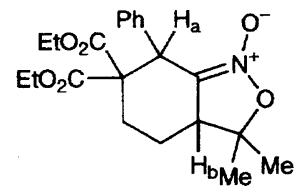

20

${ }^{4} J_{\mathrm{Ha}-\mathrm{Hb}}($ cis $) \approx 0 \mathrm{~Hz}$

${ }^{4} J_{\mathrm{Ha}-\mathrm{Hb}}($ trans $)=3.3 \mathrm{~Hz}$

Fig. 1. Comparison of ${ }^{4} J_{\mathrm{H}-\mathrm{H}}$.

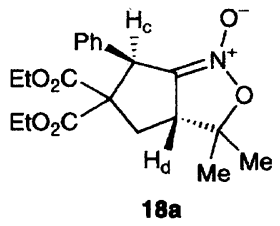

${ }^{4} J_{\mathrm{Hc}-\mathrm{Hd}}($ trans $)=3.4 \mathrm{~Hz}$ 

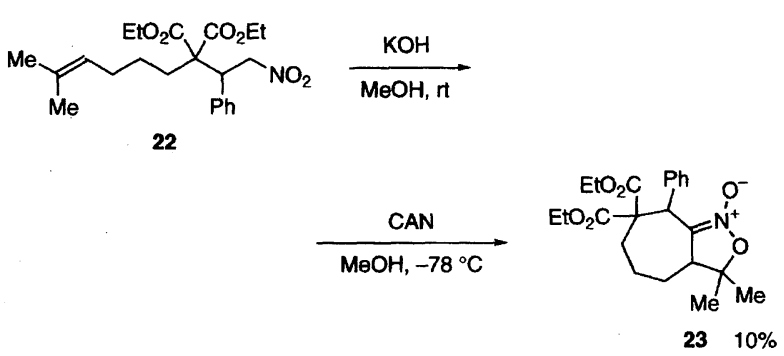

(4)

It is known that six-membered ring formation is not favorable in the radical cyclization reaction mediated by tributylstannane, because of the relatively small rate constant of the cyclization of an intermediate radical (e.g. 6-heptenyl radical) compared to that of its hydrogen abstraction from the stannane. ${ }^{22)}$ According to the above results, on the contrary, the intramolecular cyclization of the 1-nitroalkyl radical is found to be a useful method for the construction of a sixmembered ring as well as a five-membered ring. So this method is applied for the construction of decalin skeleton from a cyclohexene derivative 29.

The precursor 29 for the cyclization was expected to be prepared by carbon chain elongation of an allylic alcohol 24. To introduce a side chain to 24 , the model reaction with the silyl enol ether 2 was examined in the presence of various Lewis acids. The results are summarized in Table 4, which shows that the use of trifluoroborane-diethyl ether (1/1) affords the expected product 25 in high yield (Entries 1,2 , and 3). The product 25 was obtained in comparable yield even with a catalytic amount of trifluoroborane-diethyl ether (1/1) (Entries 4 and 5).

By using this carbon-carbon bond forming reaction, the precursor 29 was prepared in 4 steps starting from 3-methyl2-cyclohexen-1-one (Scheme 3).

When the precursor 29 was treated with potassium hydroxide followed by oxidation with CAN, the cyclized product 30 was obtained as a single stereoisomer in $62 \%$ yield (Eq. 5).

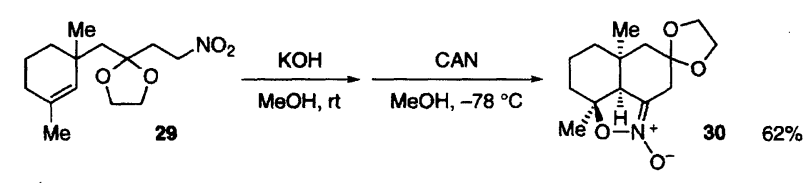

The relative configuration was determined by the differential NOE experiment (Fig. 2). This revealed that cis-decalin was constructed by this radical cyclization.

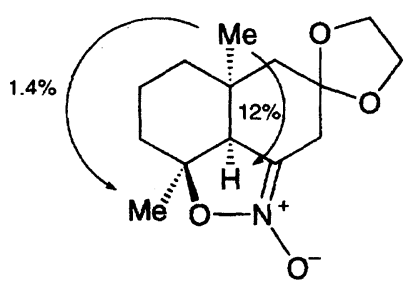

Fig. 2. Observed NOE for the compound 30.

Table 4. Reaction of $\mathbf{2 4}$ with Silyl Enol Ether Catalyzed by Lewis Acid

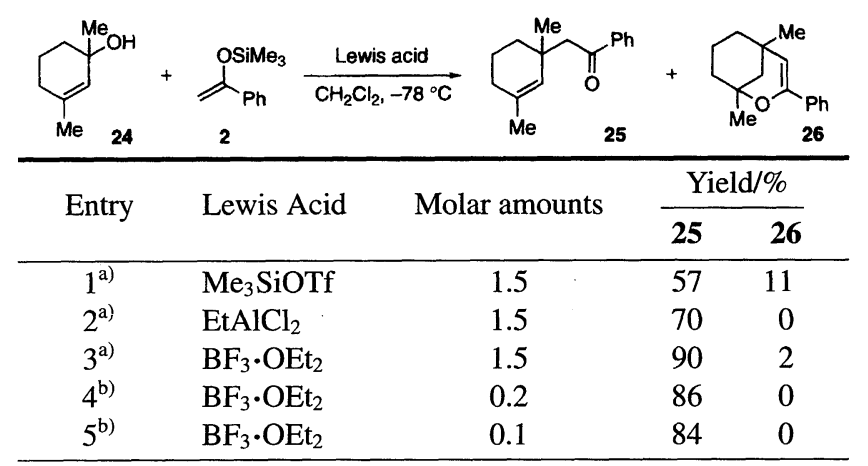

a) 1.5 molar amounts of the silyl enol ether 2 were employed.

b) 1.1 molar amounts of the silyl enol ether 2 were employed.

The aci-nitro ester $\mathbf{3 0}$ was converted quantitatively into a hydroxy ketone 31 by ozonolysis (Eq. 6), which would be a useful intermediate for the synthesis of cis-decalin derivatives.

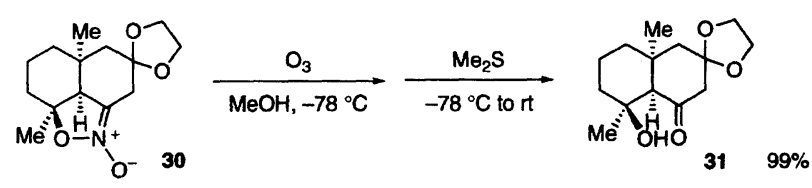

In conclusion, oxidation of potassium salt of 1-aci-nitroalkanes with CAN generates 1-nitroalkyl radicals which react with various electron-rich olefins to afford the corresponding addition products. This radical addition reaction is also utilized for the intramolecular cyclization. That is, even sixmembered fused ring systems, which are difficult to obtain by the conventional radical cyclization by using stannane, are constructed in good yield as well as five-membered ring systems.

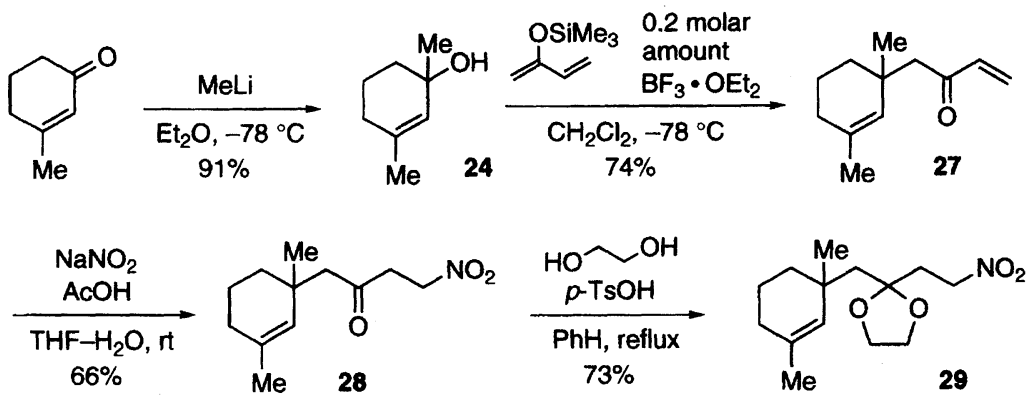

Scheme 3. Preparation of the precursor for the decalin skeleton. 


\section{Experimental}

General. ${ }^{1} \mathrm{H}$ NMR $(500 \mathrm{MHz})$ and ${ }^{13} \mathrm{C} \mathrm{NMR}(125 \mathrm{MHz})$ spectra were recorded on a Bruker AM 500 spectrometer in $\mathrm{CDCl}_{3}$ solutions using $\mathrm{CHCl}_{3}$ (for ${ }^{1} \mathrm{H}, \delta=7.24$ ) and $\mathrm{CDCl}_{3}$ (for ${ }^{13} \mathrm{C}$, $\delta=77.00$ ) as an internal standard. IR spectra were recorded on a Horiba FT 300-S spectrophotometer. High-resolution mass spectra were obtained with a JEOL JMS-SX102A mass spectrometer at an ionization energy of $70 \mathrm{eV}$. X-Ray diffraction intensities were collected on a Rigaku AFC-7R goniometer with graphite monochromated $\operatorname{Mo} K \alpha(\lambda=0.71069 \AA)$ radiation. The melting points were uncorrected. Elemental analyses were carried out at The Elemental Analysis Laboratory, Department of Chemistry, Faculty of Science, The University of Tokyo. Methanol was distilled from magnesium methoxide and stored under argon atmosphere. Dichloromethane was distilled from $\mathrm{P}_{2} \mathrm{O}_{5}$, then from $\mathrm{CaH}_{2}$, and dried over Molecular Sieves $4 \AA$. CAN (Kanto Chemical Co., Inc., guaranteed grade) was dried under vacuum at $80{ }^{\circ} \mathrm{C}$ for $10-12 \mathrm{~h}$ before use. $\left[\mathrm{Fe}\left(\eta^{5}\right.\right.$ $\left.\left.\mathrm{C}_{5} \mathrm{H}_{5}\right)_{2}\right] \mathrm{PF}_{6},{ }^{23)} \mathrm{Mn}(\text { pic })_{3},{ }^{24)}$ and $\mathrm{Ag}(\text { pic })_{2}{ }^{25)}$ were prepared according to the literature procedure. Preparative TLC was performed on a silica gel (Wakogel B-5F).

Typical Procedure for the Reaction of Nitroalkanes with Olefins. To a solution of CAN (119.5 $\mathrm{mg}, 0.218 \mathrm{mmol})$ in methanol $(1 \mathrm{ml})$ was added a solution of (1-trimethylsiloxyethenyl)benzene (2) $(18.6 \mathrm{mg}, 0.0981 \mathrm{mmol})$ in methanol $(1.5 \mathrm{ml})$ at $-78{ }^{\circ} \mathrm{C}$ under an argon atmosphere. At the same temperature, a mixture of $\mathrm{KOH}\left(1.05 \mathrm{~mol} \mathrm{dm}^{-3}\right.$ in methanol, $0.15 \mathrm{ml}, 0.158$ $\mathrm{mmol}$ ) and 1-nitro-4-phenylbutane (1a) $(20.8 \mathrm{mg}, 0.116 \mathrm{mmol})$ in methanol $(1.5 \mathrm{ml})$ was gradually added. The orange-yellow solution immediately turned dark brown and the color faded to pale yellow within 4 to $5 \mathrm{~min}$. After $15 \mathrm{~min}$, the reaction was quenched by adding $0.1 \mathrm{~mol} \mathrm{dm}^{-3}$ aqueous $\mathrm{Na}_{2} \mathrm{~S}_{2} \mathrm{O}_{3}$ (a few drops) and water. The mixture was extracted with dichloromethane $(10 \mathrm{ml} \times 4)$ and the combined extracts were dried over anhydrous $\mathrm{Na}_{2} \mathrm{SO}_{4}$. After evaporation of the solvent, the residue was treated with triethylamine (ca. $0.2 \mathrm{ml}$ ) in methanol at ambient temperature for $20 \mathrm{~min}$. The reaction mixture was diluted with water and acidified with 1 mol dm ${ }^{-3}$ aqueous $\mathrm{HCl}$. After the extraction and evaporation in the same way, chromatographic purification afforded 1,6-diphenyl2-hexen-1-one (4a) (24.0 mg, $0.0959 \mathrm{mmol}, 99 \%$ ).

A large scale run (1a, $208.3 \mathrm{mg}, 1.16 \mathrm{mmol} ; \mathbf{2}, 188.6 \mathrm{mg}, 0.981$ mmol; 1a, KOH soln, $1.5 \mathrm{ml}, 1.58 \mathrm{mmol}$; CAN, $1.21 \mathrm{~g}, 2.21 \mathrm{mmol})$ afforded almost the same result (4a, $234.1 \mathrm{mg}, 0.935 \mathrm{mmol}, 95 \%$ yield).

The spectral data of the products are as follows:

(E)-1,6-Diphenyl-2-hexen-1-one (4a): Colorless oil; IR(neat) 3028, 2931, 1670, 1620, 1290, 1225, 748, $696 \mathrm{~cm}^{-1}$; ${ }^{1} \mathrm{HNMR}$ $\delta=1.83-1.89(2 \mathrm{H}, \mathrm{m}), 2.32-2.37(2 \mathrm{H}, \mathrm{m}), 2.66-2.69(2 \mathrm{H}, \mathrm{m}$ $(\mathrm{t}-$ like $)), 6.88\left(1 \mathrm{H}, \mathrm{dt}, J_{\mathrm{d}}=15.4, J_{\mathrm{t}}=1.4 \mathrm{~Hz}\right), 7.07\left(1 \mathrm{H}, \mathrm{dt}, J_{\mathrm{d}}=15.4\right.$, $\left.J_{\mathrm{t}}=6.9 \mathrm{~Hz}\right), 7.17-7.20(3 \mathrm{H}, \mathrm{m}), 7.27-7.30(2 \mathrm{H}, \mathrm{m}(\mathrm{t}-$ like $))$, $7.44-7.47(2 \mathrm{H}, \mathrm{m}(\mathrm{t}-\mathrm{like})), 7.52-7.55(1 \mathrm{H}, \mathrm{m}$ (t-like)), 7.90$7.92\left(2 \mathrm{H}, \mathrm{m}\right.$ (d-like)); ${ }^{13} \mathrm{C}$ NMR $\delta=29.75,32.22,35.32,125.93$, $126.18,128.38,128.42,128.49,128.49,132.59,137.92,141.66$, 149.35, 190.80. Found: C, 86.06; H, 7.32\%. Calcd for $\mathrm{C}_{18} \mathrm{H}_{18} \mathrm{O}$ : C, $86.36 ; \mathrm{H}, 7.25 \%$.

(Z)-4-Nitro-1,8-diphenyl-4-octene (5): Colorless oil; IR (neat) 3026, 2937, 1518, 1454, 1335, 748, $700 \mathrm{~cm}^{-1}$; ${ }^{1} \mathrm{HNMR}$ $\delta=1.76-1.84(4 \mathrm{H}, \mathrm{m}), 2.11-2.16(2 \mathrm{H}, \mathrm{m}), 2.53-2.56(2 \mathrm{H}, \mathrm{m})$, $2.61-2.66(4 \mathrm{H}, \mathrm{m}), 7.09(1 \mathrm{H}, \mathrm{t}, J=7.9 \mathrm{~Hz}), 7.13-7.22(6 \mathrm{H}$, m), 7.26-7.31 (4H, m); ${ }^{13} \mathrm{C} \mathrm{NMR} \delta=25.91,27.23,29.30,29.93$, $35.25,35.25,126.04,126.13,128.30,128.34,128.41,128.47$, 136.07, 141.02, 141.16, 151.76. HRMS: $\mathrm{m} / \mathrm{z}$ 309.1714. Calcd for $\mathrm{C}_{20} \mathrm{H}_{23} \mathrm{NO}_{2}: \mathrm{M}, 309.1729$.

(E)-9-Phenyl-5-nonen-4-one (7a): Colorless oil; IR (neat) 2933, 1693, 1672, 1629, 1456, 978, 746, $700 \mathrm{~cm}^{-1} ;{ }^{1} \mathrm{HNMR}$ $\delta=0.92(3 \mathrm{H}, \mathrm{t}, J=7.4 \mathrm{~Hz}), 1.58-1.65(2 \mathrm{H}, \mathrm{m}), 1.76-1.82(2 \mathrm{H}$, m), $2.20-2.25(2 \mathrm{H}, \mathrm{m}), 2.48(2 \mathrm{H}, \mathrm{t}, J=7.4 \mathrm{~Hz}), 2.63(2 \mathrm{H}, \mathrm{t}, J=7.6$ $\mathrm{Hz}), 6.08\left(1 \mathrm{H}, \mathrm{dt}, J_{\mathrm{d}}=15.8, J_{\mathrm{t}}=1.5 \mathrm{~Hz}\right), 6.80\left(1 \mathrm{H}, \mathrm{dt}, J_{\mathrm{d}}=15.8\right.$, $\left.J_{\mathrm{t}}=6.9 \mathrm{~Hz}\right), 7.14-7.19(3 \mathrm{H}, \mathrm{m}), 7.25-7.28(2 \mathrm{H}, \mathrm{m}):{ }^{13} \mathrm{CNMR} \delta=$ 13.81, 17.72, 29.70, 31.84, 35.28, 42.04, 125.94, 128.38, 128.41, 130.62, 141.65, 146.62, 200.75. HRMS: $\mathrm{m} / \mathrm{z}$ 216.1509. Calcd for $\mathrm{C}_{15} \mathrm{H}_{20} \mathrm{O}: \mathrm{M}, 216.1514$.

(E)-1,8-Diphenyl-4-octen-3-one (8a): Colorless oil; IR (neat) 3026, 2931, 1693, 1672, 1630, 1495, 1452, 978, 746, $700 \mathrm{~cm}^{-1}$; ${ }^{1} \mathrm{HNMR} \delta=1.74-1.80(2 \mathrm{H}, \mathrm{m}), 2.19-2.24(2 \mathrm{H}, \mathrm{m}), 2.60-2.63$ (2H, m (t-like)), 2.82-2.86 (2H, m), 2.90-2.94 (2H, m), 6.09 $\left(1 \mathrm{H}, \mathrm{dt}, J_{\mathrm{d}}=15.8, J_{\mathrm{t}}=1.4 \mathrm{~Hz}\right), 6.80\left(1 \mathrm{H}, \mathrm{dt}, J_{\mathrm{d}}=15.8, J_{\mathrm{t}}=6.9 \mathrm{~Hz}\right)$, $7.14-7.21(6 \mathrm{H}, \mathrm{m}), 7.25-7.28(4 \mathrm{H}, \mathrm{m}) ;{ }^{13} \mathrm{CNMR} \delta=29.63$, $30.07,31.85,35.24,41.66,125.94,126.05,128.34,128.38,128.40$, $128.45,130.53,141.25,141.59,147.08,199.45$. HRMS: $\mathrm{m} / \mathrm{z}$ 278.1665. Calcd for $\mathrm{C}_{20} \mathrm{H}_{22} \mathrm{O}: \mathrm{M}, 278.1671$.

(E)-2-Methyl-1,6-diphenyl-2-hexen-1-one (9a): Colorless oil; IR (neat) 3026, 2931, 1645, 1598, 1448, 1315, 1282, 748, 704 $\mathrm{cm}^{-1} ;{ }^{1} \mathrm{H}$ NMR $\delta=1.73-1.79(2 \mathrm{H}, \mathrm{m}), 1.93(3 \mathrm{H}, \mathrm{d}, J=0.73 \mathrm{~Hz})$, 2.27-2.32 (2H, m), 2.62-2.65 (2H, m (t-like)), 6.27-6.30 (1H, m), 7.14-7.19 (3H, m), 7.25-7.28 (2H, m), 7.38-7.41 (2H, m (t-like)), 7.46-7.50 (1H, m (t-like)), 7.59-7.61 (2H, m (d-like)); ${ }^{13} \mathrm{CNMR} \delta=12.50,28.62,30.25,35.56,125.93,128.01,128.36$, 128.37, 129.24, 131.32, 136.77, 138.72, 141.67, 146.18, 198.99 . HRMS: $m / z$ 264.1513. Calcd for $\mathrm{C}_{19} \mathrm{H}_{20} \mathrm{O}: \mathrm{M}, 264.1514$.

The configuration of this compound was determined as $E$, since a cross peak was observed between the protons of methyl group and those at 4-position by NOESY experiment.

2,2-Dimethyl-3-nitro-1,6-diphenyl-1-hexanone (10a): Colorless oil; IR (neat) 2939, 1678, 1549, 1462, 1367, 1257, 960 , $700 \mathrm{~cm}^{-1} ;{ }^{1} \mathrm{HNMR} \delta=1.37(3 \mathrm{H}, \mathrm{s}), 1.42(3 \mathrm{H}, \mathrm{s}), 1.53-1.67$ $(3 \mathrm{H}, \mathrm{m}), 2.11-2.15(1 \mathrm{H}, \mathrm{m}), 2.54-2.61(2 \mathrm{H}, \mathrm{m}), 5.17(1 \mathrm{H}, \mathrm{dd}$, $J=1.9,11.3 \mathrm{~Hz}), 7.09-7.11(2 \mathrm{H}, \mathrm{m}$ (d-like) $), 7.15-7.18(1 \mathrm{H}, \mathrm{m}$ (t-like)), 7.23-7.26 (2H, m (t-like)), 7.39-7.42 (2H, m (t-like)), 7.47-7.50 (1H, m (t-like)), 7.53-7.54 (2H, m (d-like)); ${ }^{13} \mathrm{C} \mathrm{NMR}$ $\delta=20.74,23.83,28.21,28.91,34.90,50.72,93.41,126.05,127.48$, $128.29,128.40,128.43,131.43,137.89,141.02,205.54$. HRMS: $m / z$ 279.1770. Calcd for $\mathrm{C}_{20} \mathrm{H}_{23} \mathrm{O}: \mathrm{M}-\mathrm{NO}_{2}, 279.1749$.

(E)-2-(4-Phenylbutylidene)cyclopentanone (11a): Colorless oil; IR (neat) 3026, 2935, 1718, 1649, 1454, 1232, 1203, 746, $700 \mathrm{~cm}^{-1} ;{ }^{1} \mathrm{H}$ NMR $\delta=1.75-1.82(2 \mathrm{H}, \mathrm{m}), 1.87-1.94(2 \mathrm{H}, \mathrm{m})$, $2.13-2.18(2 \mathrm{H}, \mathrm{m}), 2.31(2 \mathrm{H}, \mathrm{t}, J=7.8 \mathrm{~Hz}), 2.50-2.54(2 \mathrm{H}, \mathrm{m})$, 2.60-2.64 (2H, m (t-like)), 6.53-6.57 (1H, m), 7.14-7.18 (3H, m), 7.26-7.28 (2H, m); ${ }^{13} \mathrm{CNMR} \delta=19.77,26.75,29.07,29.93$, $35.40,38.59,125.88,128.34,128.39,135.63,137.60,141.74$, 207.19. HRMS: $m / z$ 214.1355. Calcd for $\mathrm{C}_{15} \mathrm{H}_{18} \mathrm{O}: \mathrm{M}, 214.1358$.

(E)-2-(4-Phenylbutylidene)cyclohexanone (12a): Colorless oil; IR (neat) $3026,2935,1718,1685,1610,1454,748,700 \mathrm{~cm}^{-1}$; ${ }^{1} \mathrm{H} N M R \quad \delta=1.69-1.73(2 \mathrm{H}, \mathrm{m}), 1.74-1.78(2 \mathrm{H}, \mathrm{m}), 1.80-1.85$ $(2 \mathrm{H}, \mathrm{m}), 2.09-2.13(2 \mathrm{H}, \mathrm{m}), 2.39-2.43(4 \mathrm{H}, \mathrm{m}), 2.60-2.63$ $(2 \mathrm{H}, \mathrm{m}), 6.61-6.65(1 \mathrm{H}, \mathrm{m}), 7.12-7.18(3 \mathrm{H}, \mathrm{m}), 7.24-7.29(2 \mathrm{H}$, $\mathrm{m}) ;{ }^{13} \mathrm{CNMR} \delta=23.31,23.57,26.70,27.20,30.01,35.45,40.15$, 125.84, 128.32, 128.39, 136.51, 138.99, 141.83, 201.09. HRMS: $\mathrm{m} / \mathrm{z}$ 228.1520. Calcd for $\mathrm{C}_{16} \mathrm{H}_{20} \mathrm{O}: \mathrm{M}, 228.1514$.

1,1-Dimethoxy-3-nitro-1,6-diphenylhexane (13a): Colorless crystals; mp $62.0-63.0^{\circ} \mathrm{C}$ (hexane); IR (KBr) 2945, 1552, 1549, $1450,1338,1286,1184,1134,1049,768,704 \mathrm{~cm}^{-1} ;{ }^{1} \mathrm{HNMR}$ $\delta=1.40-1.46(3 \mathrm{H}, \mathrm{m}), 1.74-1.80(1 \mathrm{H}, \mathrm{m}), 2.01(1 \mathrm{H}, \mathrm{dd}, J=3.1$, $15.5 \mathrm{~Hz}), 2.38-2.47(2 \mathrm{H}, \mathrm{m}), 2.89(1 \mathrm{H}, \mathrm{dd}, J=8.7,15.5 \mathrm{~Hz})$, 
$3.07(3 \mathrm{H}, \mathrm{s}), 3.14(3 \mathrm{H}, \mathrm{s}), 4.17-4.22(1 \mathrm{H}, \mathrm{m}), 6.98-7.00(2 \mathrm{H}, \mathrm{m}$ (d-like)), 7.14-7.19 (1H, m (t-like)), 7.21-7.24 (2H, m), 7.31$7.34(1 \mathrm{H}, \mathrm{m}), 7.35-7.39(2 \mathrm{H}, \mathrm{m}), 7.41-7.44(2 \mathrm{H}, \mathrm{m}) ;{ }^{13} \mathrm{CNMR}$ $\delta=26.91,34.06,34.52,40.49,48.85,49.04,84.17,101.94,125.97$, $126.86,128.24,128.36,128.36,128.40,139.12,140.88$. Found: C, 69.88; H, 7.30; N, 4.14\%. Calcd for $\mathrm{C}_{20} \mathrm{H}_{25} \mathrm{NO}_{4}: \mathrm{C}, 69.95 ; \mathrm{H}$, $7.34 ; \mathrm{N}, 4.08 \%$.

2-Methoxy-4-nitro-2,7-diphenylheptane (14a): One of the two diastereomers $(68: 32)$ could be isolated in pure form by TLC. Colorless oil; IR (neat) 2941, 1550, 1452, 1375, 1169, 1074, 702 $\mathrm{cm}^{-1} ;{ }^{1} \mathrm{H}$ NMR $\delta=1.47-1.53(3 \mathrm{H}, \mathrm{m}), 1.51(3 \mathrm{H}, \mathrm{s}), 1.77-1.81$ $(1 \mathrm{H}, \mathrm{m}), 1.84(1 \mathrm{H}, \mathrm{dd}, J=2.0,15.4 \mathrm{~Hz}), 2.46-2.51(2 \mathrm{H}, \mathrm{m})$, $2.63(1 \mathrm{H}, \mathrm{dd}, J=9.0,15.4 \mathrm{~Hz}), 3.07(3 \mathrm{H}, \mathrm{s}), 4.61-4.62(1 \mathrm{H}, \mathrm{m})$, 7.02-7.03 (2H, m (d-like)), 7.13-7.16 (1H, m (t-like)), 7.20$7.22(2 \mathrm{H}, \mathrm{m}$ (d-like) ), 7.25-7.28 (1H, m (t-like)), 7.31-7.37 (4H, m); ${ }^{13}$ CNMR $\delta=22.50,27.10,34.70,34.88,46.87,50.53,77.87$, $84.16,125.84,125.97,127.27,128.32,128.38,128.48,141.08$, 143.95. Found: $\mathrm{C}, 73.50 ; \mathrm{H}, 7.59 ; \mathrm{N}, 4.52 \%$. Calcd for $\mathrm{C}_{20} \mathrm{H}_{25} \mathrm{NO}_{3}$ : C, 73.37; H, 7.70; N, 4.28\%.

2-Hydroxy-2,7-diphenyl-4-heptanone (15a): Colorless oil; IR (neat) 3471, 3026, 2933, 1697, 1494, 1450, 1375, 764, 748, 702 $\mathrm{cm}^{-1} ;{ }^{1} \mathrm{H} \mathrm{NMR} \delta=1.49(3 \mathrm{H}, \mathrm{s}), 1.74-1.80(2 \mathrm{H}, \mathrm{m}), 2.24(1 \mathrm{H}, \mathrm{dt}$, $\left.J_{\mathrm{d}}=17.4, J_{\mathrm{t}}=7.2 \mathrm{~Hz}\right), 2.34\left(1 \mathrm{H}, \mathrm{dt}, J_{\mathrm{d}}=17.4, J_{\mathrm{t}}=7.2 \mathrm{~Hz}\right), 2.42-2.52$ $(2 \mathrm{H}, \mathrm{m}), 2.76(1 \mathrm{H}, \mathrm{d}, J=16.8 \mathrm{~Hz}), 3.10(1 \mathrm{H}, \mathrm{d}, J=16.8 \mathrm{~Hz}), 4.61$ (1H, s), 7.05-7.07 (2H, m (d-like)), 7.15-7.18 (1H, m (t-like)), $7.19-7.26(3 \mathrm{H}, \mathrm{m}), 7.29-7.32(2 \mathrm{H}, \mathrm{m}$ (t-like) $), 7.38-7.39(2 \mathrm{H}$, m (d-like)); ${ }^{13}$ C NMR $\delta=24.51,30.67,34.72,43.63,53.23,73.35$, $124.31,125.98,126.71,128.26,128.38,128.39,141.26,147.22$, 212.64. HRMS: $m / z$ 282.1600. Calcd for $\mathrm{C}_{19} \mathrm{H}_{22} \mathrm{O}_{2}$ : M, 282.1620.

4-Nitro-7-phenyl-1-heptene (16a): Colorless oil; IR (neat) 2929, 1550, 1454, 1373, 928, 750, $700 \mathrm{~cm}^{-1}$; ${ }^{1} \mathrm{HNMR} \delta=$ $1.62-1.68(2 \mathrm{H}, \mathrm{m}), 1.71-1.78(1 \mathrm{H}, \mathrm{m}), 1.95-2.03(1 \mathrm{H}, \mathrm{m})$, $2.42-2.48(1 \mathrm{H}, \mathrm{m}), 2.61-2.67(3 \mathrm{H}, \mathrm{m}), 4.47-4.52(1 \mathrm{H}, \mathrm{m})$, $5.10-5.13(2 \mathrm{H}, \mathrm{m}), 5.62-5.70(1 \mathrm{H}, \mathrm{m}), 7.12-7.13(2 \mathrm{H}, \mathrm{m})$, $7.16-7.19(1 \mathrm{H}, \mathrm{m}), 7.25-7.28(2 \mathrm{H}, \mathrm{m}) ;{ }^{13} \mathrm{CNMR} \delta=27.35$, $32.69,35.03,37.90,88.02,119.49,126.11,128.32,128.47,131.43$, 141.03. Found: C, $70.91 ; \mathrm{H}, 7.67 ; \mathrm{N}, 6.60 \%$. Calcd for $\mathrm{C}_{13} \mathrm{H}_{17} \mathrm{NO}_{2}$ : C, $71.21 ; \mathrm{H}, 7.81 ; \mathrm{N}, 6.39 \%$.

(E)-7-Oxo-7-phenyl-5-heptenyl Acetate (4b): Colorless oil; IR (neat) 2945, 1738, 1670, 1622, 1242, $696 \mathrm{~cm}^{-1} ;{ }^{1} \mathrm{H}$ NMR $\delta=1.57-1.61(2 \mathrm{H}, \mathrm{m}), 1.65-1.69(2 \mathrm{H}, \mathrm{m}), 2.03(3 \mathrm{H}, \mathrm{s}), 2.32-$ $2.36(2 \mathrm{H}, \mathrm{m}), 4.07(2 \mathrm{H}, \mathrm{t}, J=6.5 \mathrm{~Hz}), 6.87\left(1 \mathrm{H}, \mathrm{dt}, J_{\mathrm{d}}=15.4\right.$, $\left.J_{\mathrm{t}}=1.4 \mathrm{~Hz}\right), 7.02\left(1 \mathrm{H}, \mathrm{dt}, J_{\mathrm{d}}=15.4, J_{\mathrm{t}}=6.9 \mathrm{~Hz}\right), 7.43-7.46(2 \mathrm{H}$, m (t-like)), 7.52-7.55 (1H, m (t-like)), 7.89-7.91 (2H, m (dlike)); ${ }^{13} \mathrm{CNMR} \delta=20.94,24.57,28.14,32.27,64.02,126.26$, $128.49,128.50,132.64,137.88,148.90,171.12,190.72$. HRMS: $\mathrm{m} / \mathrm{z}$ 246.1260. Calcd for $\mathrm{C}_{15} \mathrm{H}_{18} \mathrm{O}_{3}: \mathrm{M}, 246.1256$.

(E)-7-Oxo-5-decenyl Acetate (7b): Colorless oil; IR (neat) 2962, 2935, 1739, 1672, 1631, 1367, 1240, $1045 \mathrm{~cm}^{-1}$; ${ }^{1} \mathrm{H}$ NMR $\delta=0.91(3 \mathrm{H}, \mathrm{t}, J=7.4 \mathrm{~Hz}), 1.49-1.57(2 \mathrm{H}, \mathrm{m}), 1.58-1.68(4 \mathrm{H}$, m), $2.02(3 \mathrm{H}, \mathrm{s}), 2.20-2.24(2 \mathrm{H}, \mathrm{m}), 2.48(2 \mathrm{H}, \mathrm{t}, J=7.2 \mathrm{~Hz})$, $4.04(2 \mathrm{H}, \mathrm{t}, J=6.5 \mathrm{~Hz}), 6.08\left(1 \mathrm{H}, \mathrm{dt}, J_{\mathrm{d}}=15.9, J=1.4 \mathrm{~Hz}\right), 6.78$ $\left(1 \mathrm{H}, \mathrm{dt}, J_{\mathrm{d}}=15.4, J_{\mathrm{t}}=6.9 \mathrm{~Hz}\right) ;{ }^{13} \mathrm{C}$ NMR $\delta=13.78,17.66,20.93$, 24.50, 28.10, 31.88, 42.08, 64.00, 130.66, 146.15, 171.12, 200.65 . HRMS: $m / z$ 212.1432. Calcd for $\mathrm{C}_{12} \mathrm{H}_{20} \mathrm{O}_{3}$ : M, 212.1412.

(E)-6-Methyl-7-oxo-7-phenyl-5-heptenyl Acetate (9b): Colorless oil; IR (neat) 2937, 1738, 1645, 1446, 1365, 1240, 1045, 708 $\mathrm{cm}^{-1} ;{ }^{1} \mathrm{HNMR} \delta=1.45-1.51(2 \mathrm{H}, \mathrm{m}), 1.61-1.67(2 \mathrm{H}, \mathrm{m}), 1.94$ $(3 \mathrm{H}, \mathrm{s}), 2.01(3 \mathrm{H}, \mathrm{s}), 2.27-2.32(2 \mathrm{H}, \mathrm{m}$ (q-like) $), 4.04(2 \mathrm{H}, \mathrm{t}, J=6.5$ $\mathrm{Hz}), 6.24(1 \mathrm{H}, \mathrm{t}, J=7.3 \mathrm{~Hz}), 7.37-7.41(2 \mathrm{H}, \mathrm{m}$ (t-like)), 7.46-7.49 (1H, m (t-like)), 7.58-7.60 (2H, m (t-like)); ${ }^{13} \mathrm{C} \mathrm{NMR} \delta=12.51$, $20.92,25.01,28.33,28.63,64.04,128.02,129.23,131.36,136.88$,
138.61, 145.58, 171.10, 198.91. HRMS: $\mathrm{m} / \mathrm{z}$ 260.1427. Calcd for $\mathrm{C}_{16} \mathrm{H}_{20} \mathrm{O}_{3}: \mathrm{M}, 260.1412$.

$(E)-5$ - $(t$-Butyl)dimethylsiloxy-1-phenyl-2-penten-1-one (4c): Colorless oil; IR $\left(\mathrm{CCl}_{4}\right)$ 2931, 1674, 1626, 1228, $1101 \mathrm{~cm}^{-1}$; ${ }^{1} \mathrm{HNMR} \delta=0.04(6 \mathrm{H}, \mathrm{s}), 0.87(9 \mathrm{H}, \mathrm{s}), 2.49-2.53(2 \mathrm{H}, \mathrm{m}), 3.78$ $(2 \mathrm{H}, \mathrm{t}, J=6.4 \mathrm{~Hz}), 6.91(1 \mathrm{H}, \mathrm{d}, J=15.6 \mathrm{~Hz}), 7.02\left(1 \mathrm{H}, \mathrm{dt}, J_{\mathrm{d}}=15.6\right.$, $\left.J_{\mathrm{t}}=6.9 \mathrm{~Hz}\right), 7.43-7.46(2 \mathrm{H}, \mathrm{m}(\mathrm{t}-\mathrm{like})), 7.52-7.55(1 \mathrm{H}, \mathrm{m}(\mathrm{t}-$ like $))$, 7.89-7.91 (2H, m (d-like)); ${ }^{13} \mathrm{CNMR} \delta=-5.37,25.84,36.28$, $53.36,61.59,127.59,128.33,128.46,128.53,132.56,146.44$, 190.83. HRMS: $m / z$ 233.0990. Calcd for $\mathrm{C}_{13} \mathrm{H}_{17} \mathrm{O}_{2} \mathrm{Si}: \mathrm{M}-\mathrm{C}_{4} \mathrm{H}_{9}$ $(t-\mathrm{Bu}), 233.0998$.

3-Methyl-1-phenyl-2-buten-1-one (4d): ${ }^{26)} \quad$ Colorless oil; IR (neat) 3060, 2912, 1660,1614, 1448, 1248, 1011, $700 \mathrm{~cm}^{-1}$; ${ }^{1} \mathrm{H}$ NMR $\delta=2.00(3 \mathrm{H}, \mathrm{s}), 2.19(3 \mathrm{H}, \mathrm{s}), 6.72-6.73(1 \mathrm{H}, \mathrm{m}), 7.41-$ $7.44(2 \mathrm{H}, \mathrm{m}), 7.48-7.52(1 \mathrm{H}, \mathrm{m}), 7.90-7.91(2 \mathrm{H}, \mathrm{m}) ;{ }^{13} \mathrm{CNMR}$ $\delta=21.15,27.96,121.19,128.16,128.41,132.24,139.26,156.64$, 191.53 .

5-Methyl-1-phenyl-4-hexen-3-one (8d): Colorless oil; IR(neat) 3060, 2931, 1687, 1622, 1446, 1111, $700 \mathrm{~cm}^{-1} ;{ }^{1} \mathrm{HNMR}$ $\delta=1.86(3 \mathrm{H}, \mathrm{d}, J=1.0 \mathrm{~Hz}), 2.14(3 \mathrm{H}, \mathrm{d}, J=0.90 \mathrm{~Hz}), 2.71-$ $2.74(2 \mathrm{H}, \mathrm{m}(\mathrm{t}-$ like $)), 2.88-2.91(2 \mathrm{H}, \mathrm{m}(\mathrm{t}-$ like $)), 6.04-6.05$ $(1 \mathrm{H}, \mathrm{m}), 7.15-7.18(3 \mathrm{H}, \mathrm{m}), 7.24-7.27(2 \mathrm{H}, \mathrm{m}) ;{ }^{13} \mathrm{CNMR}$ $\delta=20.76,27.66,30.08,45.75,123.65,125.93,128.33,128.40$, 141.47, 155.39, 199.82. HRMS: $m / z$ 188.1212. Calcd for $\mathrm{C}_{13} \mathrm{H}_{16} \mathrm{O}$ : M, 188.1201.

2-Cyclohexylidene-1-phenyl-1-ethanone (4e): Colorless oil; IR (neat) 2929, 1658, 1614, 1446, 1219, $702 \mathrm{~cm}^{-1} ;{ }^{1} \mathrm{H} N M R$ $\delta=1.59-1.65(4 \mathrm{H}, \mathrm{m}), 1.69-1.73(2 \mathrm{H}, \mathrm{m}), 2.28-2.31(2 \mathrm{H}, \mathrm{m})$, $2.74-2.76(2 \mathrm{H}, \mathrm{m}(\mathrm{t}-\mathrm{like})), 6.58(1 \mathrm{H}, \mathrm{s}) 7.41-7.44(2 \mathrm{H}, \mathrm{m})$, $7.49-7.52(1 \mathrm{H}, \mathrm{m}), 7.91-7.93(2 \mathrm{H}, \mathrm{m}) ;{ }^{13} \mathrm{CNMR} \delta=26.27$, 27.97, 28.85, 30.63, 38.39, 118.72, 128.72, 128.31, 128.37, 132.30, 139.27, 162.75, 192.36. HRMS: $m / z 200.1212$. Calcd for $\mathrm{C}_{14} \mathrm{H}_{16} \mathrm{O}$ : M, 200.1201.

1-Cyclohexylidene-4-phenyl-2-butanone (8e): Colorless oil; IR (neat) 2935, 1687, 1622, 1448, $702 \mathrm{~cm}^{-1}$; ${ }^{1} \mathrm{HNMR} \delta=$ $1.57-1.64(6 \mathrm{H}, \mathrm{m}), 2.13-2.15(2 \mathrm{H}, \mathrm{m}), 2.72-2.75(2 \mathrm{H}, \mathrm{m})$, $2.78-2.80(2 \mathrm{H}, \mathrm{m}), 2.88-2.92(2 \mathrm{H}, \mathrm{m}), 5.94(1 \mathrm{H}, \mathrm{s}), 7.15-$ $7.19(3 \mathrm{H}, \mathrm{m}), 7.24-7.27(2 \mathrm{H}, \mathrm{m}) ;{ }^{13} \mathrm{CNMR} \delta=26.23,27.89$, $28.78,30.01,30.14,38.10,45.92,120.85,125.91,128.34,128.39$, 141.46, 162.22, 200.59. HRMS: $m / z$ 228.1512. Calcd for $\mathrm{C}_{16} \mathrm{H}_{20} \mathrm{O}$ : M, 228.1514.

(E)-1,3-Diphenyl-2-propen-1-one (4f): ${ }^{27)} \quad{ }^{1} \mathrm{HNMR} \delta=$ $7.40-7.65(9 \mathrm{H}, \mathrm{m}), 7.80(1 \mathrm{H}, \mathrm{d}, J=15.7 \mathrm{~Hz}), 8.02-8.05(2 \mathrm{H}$, m (d-like)).

Typical Procedure for the Preparation of Substrates for Intramolecular Cyclization. To a suspension of sodium hydride ( $60 \%$ oil dispersion, $0.22 \mathrm{~g}, 5.5 \mathrm{mmol}$, washed with petroleum ether before use) in tetrahydrofuran $(10 \mathrm{ml})$ was added a solution of diethyl (3-methyl-2-butenyl)malonate $(1.14 \mathrm{~g}, 4.99 \mathrm{mmol})$ in tetrahydrofuran $(2 \mathrm{ml})$ at $0{ }^{\circ} \mathrm{C}$ under an argon atmosphere. After stirring at $0{ }^{\circ} \mathrm{C}$ for $15 \mathrm{~min}$, the mixture was cooled to $-78^{\circ} \mathrm{C}$, and then a solution of $((E)$-2-nitroethenyl $)$ benzene $(0.746 \mathrm{~g}, 5.00 \mathrm{mmol})$ in tetrahydrofuran $(3 \mathrm{ml})$ was added. Stirring was continued for 2 $\mathrm{h}$ at $-78^{\circ} \mathrm{C}$, and the reaction was quenched by adding $\mathrm{pH} 7$ phosphate buffer solution. The mixture was extracted with ethyl acetate $(20 \mathrm{ml} \times 4)$ and dried over anhydrous $\mathrm{Na}_{2} \mathrm{SO}_{4}$. After evaporation of the solvent, chromatographic purification of the residue afforded diethyl (3-methyl-2-butenyl)(2-nitro-1-phenylethyl)malonate (17a) $(1.09 \mathrm{~g}, 2.89 \mathrm{mmol}, 58 \%)$.

The spectral data of the products are as follows:

Diethyl (3-Methyl-2-butenyl)(2-nitro-1-phenylethyl)malonate (17a): Pale yellow oil; IR (neat) 2983, 1730, 1556, 1450, 1377, 
$1294,1227,1184,704 \mathrm{~cm}^{-1} ;{ }^{1} \mathrm{H}$ NMR $\delta=1.23(3 \mathrm{H}, \mathrm{t}, J=7.1 \mathrm{~Hz})$, $1.30(3 \mathrm{H}, \mathrm{t}, J=7.1 \mathrm{~Hz}), 1.41(3 \mathrm{H}, \mathrm{s}), 1.70(3 \mathrm{H}, \mathrm{s}), 2.20(1 \mathrm{H}, \mathrm{dd}$, $J=7.9,15.4 \mathrm{~Hz}), 2.50(1 \mathrm{H}, \mathrm{dd}, J=6.1,15.4 \mathrm{~Hz}), 4.14-4.22(3 \mathrm{H}$, $\mathrm{m}), 4.24-4.31(2 \mathrm{H}, \mathrm{m}), 4.92(1 \mathrm{H}, \mathrm{dd}, J=11.2,13.4 \mathrm{~Hz}), 5.04(1 \mathrm{H}$, br t), $5.11(1 \mathrm{H}, \mathrm{dd}, J=3.0,13.4 \mathrm{~Hz}), 7.06-7.08(2 \mathrm{H}, \mathrm{m}), 7.24$ $7.26(3 \mathrm{H}, \mathrm{m}) ;{ }^{13} \mathrm{CNMR} \delta=13.97,14.00,18.02,26.09,32.32$, $46.20,60.27,61.86,61.89,78.63,116.93,128.30,128.69,128.90$, 135.21, 136.16, 169.85, 170.12. HRMS: $\mathrm{m} / \mathrm{z}$ 378.1924. Calcd for $\mathrm{C}_{20} \mathrm{H}_{28} \mathrm{NO}_{6}: \mathrm{M}+\mathrm{H}, 378.1917$.

Diethyl (3-Methyl-2-butenyl)[2-nitro-1-(4-bromophenyl)ethyl]malonate (17b): $\quad$ Colorless crystals; $\mathrm{mp} 82.0-82.5^{\circ} \mathrm{C}$ (hexane-diethyl ether); IR (KBr) 2991, 1736, 1666, 1549, 1450, 1383, $1308,1220,1192,1173 \mathrm{~cm}^{-1} ;{ }^{1} \mathrm{HNMR} \delta=1.23(3 \mathrm{H}, \mathrm{t}, J=7.1 \mathrm{~Hz})$, $1.29(3 \mathrm{H}, \mathrm{t}, J=7.1 \mathrm{~Hz}), 1.43(3 \mathrm{H}, \mathrm{s}), 1.70(3 \mathrm{H}, \mathrm{s}), 2.19(1 \mathrm{H}, \mathrm{dd}$, $J=7.9,15.5 \mathrm{~Hz}), 2.50(1 \mathrm{H}, \mathrm{dd}, J=6.1,15.5 \mathrm{~Hz}), 4.15-4.22(3 \mathrm{H}$, m), $4.23-4.30(2 \mathrm{H}, \mathrm{m}), 4.87(1 \mathrm{H}, \mathrm{dd}, J=11.3,13.5 \mathrm{~Hz}), 5.01(1 \mathrm{H}$, br t), $5.10(1 \mathrm{H}, \mathrm{dd}, J=3.0,13.5 \mathrm{~Hz}), 6.96(2 \mathrm{H}, \mathrm{d}, J=8.5 \mathrm{~Hz}), 7.40$ $(2 \mathrm{H}, \mathrm{d}, J=8.5 \mathrm{~Hz}) ;{ }^{13} \mathrm{CNMR} \delta=13.94,14.00,18.08,26.06,32.26$, $45.61,60.04,62.01,62.01,78.27,116.62,122.46,130.58,131.85$, 134.33, 136.45, 169.67, 169.90. Found: C, 52.38; H, 5.68; N, $3.20 \%$. Calcd for $\mathrm{C}_{20} \mathrm{H}_{26} \mathrm{BrNO}_{6}$ : C, 52.64; $\mathrm{H}, 5.74 ; \mathrm{N}, 3.07 \%$.

Diethyl (4-Methyl- 3- pentenyl)(2- nitro- 1- phenylethyl)malonate (19): Pale yellow oil; IR (neat) 2979, 1728, 1556. 1450, 1377, 1221, $704 \mathrm{~cm}^{-1} ;{ }^{1} \mathrm{H}$ NMR $\delta=1.28(3 \mathrm{H}, \mathrm{t}, J=7.2 \mathrm{~Hz}), 1.29$ $(3 \mathrm{H}, \mathrm{t}, J=7.2 \mathrm{~Hz}), 1.44-1.49(1 \mathrm{H}, \mathrm{m}), 1.51(3 \mathrm{H}, \mathrm{s}), 1.61(3 \mathrm{H}$ s), $1.71-1.77(1 \mathrm{H}, \mathrm{m}), 1.86-1.91(1 \mathrm{H}, \mathrm{m}), 1.95-2.02(1 \mathrm{H}, \mathrm{m})$, $4.19(1 \mathrm{H}, \mathrm{dd}, J=3.0,11.0 \mathrm{~Hz}), 4.22-4.31(4 \mathrm{H}, \mathrm{m}), 4.90(1 \mathrm{H}$, br t), $4.94(1 \mathrm{H}, \mathrm{dd}, J=11.0,13.4 \mathrm{~Hz}), 5.05(1 \mathrm{H}, \mathrm{dd}, J=3.0,13.4$ $\mathrm{Hz}), 7.09-7.11(2 \mathrm{H}, \mathrm{m}), 7.26-7.30(3 \mathrm{H}, \mathrm{m}) ;{ }^{13} \mathrm{CNMR} \delta=13.98$, $14.07,17.58,23.04,25.59,33.82,46.78,60.20,61.81,61.95,78.84$, $122.61,128.40,128.79,128.79,132.68,135.24,169.86,170.32$. HRMS: $m / z$ 392.2052. Calcd for $\mathrm{C}_{21} \mathrm{H}_{30} \mathrm{NO}_{6}: \mathrm{M}+\mathrm{H}, 392.2073$.

Diethyl (5-Methyl-4-hexenyl)(2-nitro-1-phenylethyl)malonate (22): Colorless oil; IR (neat) 2976, 1728, 1556, 1452, 1377, $1209,704 \mathrm{~cm}^{-1}$; ${ }^{1} \mathrm{HNMR} \delta=1.44-1.22(1 \mathrm{H}, \mathrm{m}), 1.26(3 \mathrm{H}, \mathrm{t}$, $J=7.2 \mathrm{~Hz}), 1.29(3 \mathrm{H}, \mathrm{t}, J=7.2 \mathrm{~Hz}), 1.33-1.40(1 \mathrm{H}, \mathrm{m}), 1.41-$ $1.46(1 \mathrm{H}, \mathrm{m}), 1.50(3 \mathrm{H}, \mathrm{s}), 1.62(3 \mathrm{H}, \mathrm{d}, J=0.6 \mathrm{~Hz}), 1.72-1.75(1 \mathrm{H}$, $\mathrm{m}), 1.82-1.91(2 \mathrm{H}, \mathrm{m}), 4.18(1 \mathrm{H}, \mathrm{dd}, J=3.0,11.1 \mathrm{~Hz}), 4.21-$ $4.29(4 \mathrm{H}, \mathrm{m}), 4.93(1 \mathrm{H}, \mathrm{dd}, J=11.1,13.5 \mathrm{~Hz}), 4.97(1 \mathrm{H}, \mathrm{br} \mathrm{t}), 5.06$ $(1 \mathrm{H}, \mathrm{dd}, J=3.0,13.5 \mathrm{~Hz}), 7.07-7.09(2 \mathrm{H}, \mathrm{m}), 7.26-7.28(3 \mathrm{H}$, m); ${ }^{13}$ CNMR $\delta=13.98,14.06,17.59,24.40,25.63,27.84,33.26$, $46.39,60.25,61.78,61.39,78.75,123.45,128.37,128.73,128.73$, 132.28, 135.25, 169.93, 170.34. HRMS: $\mathrm{m} / \mathrm{z}$ 405.2136. Calcd for $\mathrm{C}_{22} \mathrm{H}_{31} \mathrm{NO}_{6}: \mathrm{M}, 405.2151$.

Typical Procedure for the Intramolecular Cyclization. To a solution of CAN (113.6 mg, $0.207 \mathrm{mmol})$ in methanol $(2 \mathrm{ml})$ was added a solution of potassium salt of aci-nitro compound (prepared by adding $\mathrm{KOH}\left(1.05 \mathrm{~mol} \mathrm{dm}^{-3}\right.$ in methanol, $\left.0.10 \mathrm{ml}, 0.105 \mathrm{mmol}\right)$ to a solution of diethyl (3-methyl-2-butenyl)(2-nitro-1-phenylethyl)malonate (17a) $(38.0 \mathrm{mg}, 0.101 \mathrm{mmol})$ in methanol $(2 \mathrm{ml}))$ at $-78{ }^{\circ} \mathrm{C}$ under an argon atmosphere. The orange-yellow solution immediately turned dark brown and the color faded to pale yellow within 4 to $5 \mathrm{~min}$. After $15 \mathrm{~min}$, the reaction was quenched by adding $0.1 \mathrm{~mol} \mathrm{dm}{ }^{-3}$ aqueous $\mathrm{Na}_{2} \mathrm{~S}_{2} \mathrm{O}_{3}$ (a few drops) and water. The mixture was extracted with dichloromethane $(10 \mathrm{ml} \times 4)$ and dried over anhydrous $\mathrm{Na}_{2} \mathrm{SO}_{4}$. After evaporation of the solvent, the residue was purified by TLC (hexane-ethyl acetate, $3: 1(\mathrm{v} / \mathrm{v})$ ) to afford the product $18 \mathbf{a}(29.3 \mathrm{mg}, 0.0780 \mathrm{mmol}, 77 \%)$.

The spectral data of the products are as follows:

Diethyl $\left(6 R^{*}, 3 \mathrm{a} R^{*}\right)-( \pm)-3 \mathrm{a}, 4,5,6-T e t r a h y d r o-3,3-d i m e t h y l-6-$ phenyl-3H-cyclopenta $[c]$ isooxazole-5,5-dicarboxylate 1-Oxide (18a): Colorless crystals; mp 87.0-87.5 ${ }^{\circ} \mathrm{C}$ (hexane); IR (KBr)
$2983,1753,1724,1676,1460,1369,1261,1198,1093,1095,773$ $\mathrm{cm}^{-1}$; ${ }^{1} \mathrm{H}$ NMR $\delta=0.70(3 \mathrm{H}, \mathrm{t}, J=7.1 \mathrm{~Hz}), 1.26(3 \mathrm{H}, \mathrm{t}, J=7.1$ $\mathrm{Hz}), 1.40(3 \mathrm{H}, \mathrm{s}), 1.59(3 \mathrm{H}, \mathrm{s}), 1.92(1 \mathrm{H}, \mathrm{dd}, J=11.0,12.8 \mathrm{~Hz})$ $2.54(1 \mathrm{H}, \mathrm{dd}, J=7.5,12.8 \mathrm{~Hz}), 3.29\left(1 \mathrm{H}, \mathrm{dq}, J_{\mathrm{a}}=10.7, J_{\mathrm{q}}=7.1\right.$ $\mathrm{Hz}), 3.67\left(1 \mathrm{H}, \mathrm{dq}, J_{\mathrm{d}}=10.7, J_{\mathrm{q}}=7.1 \mathrm{~Hz}\right), 4.21\left(1 \mathrm{H}, \mathrm{dq}, J_{\mathrm{d}}=10.8\right.$, $\left.J_{\mathrm{q}}=7.1 \mathrm{~Hz}\right), 4.31\left(1 \mathrm{H}, \mathrm{dq}, J_{\mathrm{d}}=10.8, J_{\mathrm{q}}=7.1 \mathrm{~Hz}\right), 4.43(1 \mathrm{H}, \mathrm{ddd}$ $J=3.4,7.5,11.0 \mathrm{~Hz}), 5.01(1 \mathrm{H}, \mathrm{d}, J=3.4 \mathrm{~Hz}), 7.19-7.27(5 \mathrm{H}$, $\mathrm{m}) ;{ }^{13} \mathrm{CNMR} \delta=13.20,13.98,22.67,26.99,32.76,47.18,57.37$, $61.65,62.15,70.98,85.20,123.89,127.88,128.41,128.61,134.68$ 169.17, 169.94. Found: C, 63.86; H, 6.55; N, 3.83\%. Calcd for $\mathrm{C}_{20} \mathrm{H}_{25} \mathrm{NO}_{6}: \mathrm{C}, 63.99 ; \mathrm{H}, 6.71 ; \mathrm{N}, 3.73 \%$.

Diethyl $\left(6 R^{*}, 3 a R^{*}\right)-( \pm)-3 a, 4,5,6-T e t r a h y d r o-3,3-d i m e t h y l-6-$ (4-bromophenyl)-3H-cyclopenta[ $[c]$ isooxazole-5,5-dicarboxylate 1-Oxide (18b): Colorless crystals; $\mathrm{mp} 138.0^{\circ} \mathrm{C}$ (hexane-diethyl ether); IR (KBr) 2976, 1751, 1728, 1674, 1263, 1230, 1188, 1061 $\mathrm{cm}^{-1} ;{ }^{1} \mathrm{HNMR} \delta=0.67(3 \mathrm{H}, \mathrm{t}, J=7.1 \mathrm{~Hz}), 1.25(3 \mathrm{H}, \mathrm{t}, J=7.1$ $\mathrm{Hz}), 1.38(3 \mathrm{H}, \mathrm{s}), 1.58(3 \mathrm{H}, \mathrm{s}), 1.91(1 \mathrm{H}, \mathrm{dd}, J=11.1,12.8 \mathrm{~Hz})$, $2.51(1 \mathrm{H}, \mathrm{dd}, J=7.5,12.8 \mathrm{~Hz}), 3.41\left(1 \mathrm{H}, \mathrm{dq}, J_{\mathrm{d}}=10.7, J_{\mathrm{q}}=7.1 \mathrm{~Hz}\right)$, $3.72\left(1 \mathrm{H}, \mathrm{dq}, J_{\mathrm{d}}=10.7, J_{\mathrm{q}}=7.1 \mathrm{~Hz}\right), 4.19\left(1 \mathrm{H}, \mathrm{dq}, J_{\mathrm{d}}=10.8, J_{\mathrm{q}}=7.1\right.$ $\mathrm{Hz}), 4.30\left(1 \mathrm{H}, \mathrm{dq}, J_{\mathrm{d}}=10.8, J_{\mathrm{q}}=7.1 \mathrm{~Hz}\right), 4.41(1 \mathrm{H}, \mathrm{ddd}, J=3.5$, $7.5,11.1 \mathrm{~Hz}), 4.94(1 \mathrm{H}, \mathrm{d}, J=3.5 \mathrm{~Hz}), 7.13(2 \mathrm{H}, \mathrm{d}, J=8.4 \mathrm{~Hz})$, $7.39(2 \mathrm{H}, \mathrm{d}, J=8.4 \mathrm{~Hz}) ;{ }^{13} \mathrm{CNMR} \delta=13.23,13.93,22.61,26.85$, $32.70,46.65,57.10,61.81,62.26,70.84,85.56,121.95,123.34$, 130.35, 131.49, 133.68, 168.94, 169.74. Found: C, 52.99; H, 5.26; $\mathrm{N}, 3.16 \%$. Calcd for $\mathrm{C}_{20} \mathrm{H}_{24} \mathrm{BrNO}_{6}: \mathrm{C}, 52.87 ; \mathrm{H}, 5.32 ; \mathrm{N}, 3.08 \%$.

Diethyl 3,3a,4,5,6,7-Hexahydro-3,3-dimethyl-7-phenylcyclohexa[c]isooxazole-6,6-dicarboxylate 1-Oxide (20): Colorless oil, obtained as a mixture of two diastereomers $(80: 20)$. In the assignment of ${ }^{13} \mathrm{CNMR}$ described below, the sign of cis and trans refers to $\left(7 R^{*}, 3 \mathrm{a} S^{*}\right)-( \pm)$ and $\left(7 R^{*}, 3 \mathrm{a} R^{*}\right)-( \pm)$-isomers respectively. IR (neat) 2978, 1730, 1649, 1454, 1371, 1291, $704 \mathrm{~cm}^{-1}$; ${ }^{1} \mathrm{H}$ NMR $\delta=0.97(3 \mathrm{H} \times 0.8, \mathrm{t}, J=7.1 \mathrm{~Hz}), 1.02(3 \mathrm{H} \times 0.2, \mathrm{t}, J=7.1$ $\mathrm{Hz}), 1.19(3 \mathrm{H} \times 0.2, \mathrm{t}, J=7.1 \mathrm{~Hz}), 1.25(3 \mathrm{H} \times 0.2, \mathrm{t}, J=7.1 \mathrm{~Hz})$, $1.28(3 \mathrm{H}, \mathrm{s}), 1.35-1.42(1 \mathrm{H} \times 0.8, \mathrm{~m}), 1.38(3 \mathrm{H} \times 0.2, \mathrm{~s}), 1.45$ $(3 \mathrm{H} \times 0.8, \mathrm{~s}), 1.71-1.76(1 \mathrm{H} \times 0.2, \mathrm{~m}), 1.86-1.90(1 \mathrm{H} \times 0.8, \mathrm{~m})$, $1.90-2.00(1 \mathrm{H} \times 0.2, \mathrm{~m}), 2.18-2.24(1 \mathrm{H} \times 0.2, \mathrm{~m}), 2.37-2.47$ $(2 \mathrm{H} \times 0.8+1 \mathrm{H} \times 0.2, \mathrm{~m}), 3.27-3.31(1 \mathrm{H} \times 0.2, \mathrm{~m}), 3.36(1 \mathrm{H} \times 0.8$, dd, $J=6.3,12.2 \mathrm{~Hz}), 3.71-3.78(1 \mathrm{H}, \mathrm{m}), 3.84-3.91(1 \mathrm{H}, \mathrm{m})$, $4.17-4.24(1 \mathrm{H}, \mathrm{m}), 4.25-4.30(1 \mathrm{H}, \mathrm{m}), 4.60(1 \mathrm{H} \times 0.2, \mathrm{~d}, J=3.3$ $\mathrm{Hz}), 4.74(1 \mathrm{H} \times 0.8, \mathrm{~s}), 7.19-7.24(5 \mathrm{H}, \mathrm{m}) ;{ }^{13} \mathrm{CNMR} \delta=13.57$ (cis), 13.62 (trans), 13.89 (trans), 14.03 (cis) 18.09 (trans), 20.61 (cis), 21.50 (trans), 22.00 (cis), 25.87 (trans), 26.41 (cis), 26.50 (trans), 26.70 (cis), 43.14 (cis), 44.70 (trans), 48.40 (trans), 50.30 (cis), 58.36 (cis), 58.60 (trans), 61.42 (cis), 61.49 (trans), 62.05 (trans), 62.11 (cis), 82.76 (cis), 83.22 (trans), 116.14 (trans), 116.36 (cis), 127.87 (trans), 127.99 (cis), 128.22 (trans), 128.59 (cis), 129.02 (cis), 129.35 (trans), 134.05 (trans), 135.44 (cis), 168.48 (cis), 170.13 (cis), 171.02 (trans). HRMS: $\mathrm{m} / \mathrm{z} 389.1833$. Calcd for $\mathrm{C}_{21} \mathrm{H}_{27} \mathrm{NO}_{6}$ : $\mathrm{M}+\mathrm{H}, 389.1838$.

Diethyl $c$-4-Isopropenyl- $t$-3-nitro- $r$-2-phenylcyclohexanedicarboxylate (21): Colorless oil; IR (neat) 2981, 1724, 1649, 1556, $1450,1369,1269,1240,1108,750,702 \mathrm{~cm}^{-1} ;{ }^{1} \mathrm{H}$ NMR $\delta=0.82$ $(3 \mathrm{H}, \mathrm{t}, J=7.1 \mathrm{~Hz}), 1.18(3 \mathrm{H}, \mathrm{t}, J=7.1 \mathrm{~Hz}), 1.57-1.66(1 \mathrm{H}, \mathrm{m}), 1.76$ $(3 \mathrm{H}, \mathrm{s}), 1.84-1.89(1 \mathrm{H}, \mathrm{m}), 2.24-2.31(1 \mathrm{H}, \mathrm{m}), 2.33-2.37(1 \mathrm{H}$, m), $2.79-2.84(1 \mathrm{H}, \mathrm{m}), 3.53(1 \mathrm{H}, \mathrm{d}, J=11.5 \mathrm{~Hz}), 3.71(1 \mathrm{H}, \mathrm{dq}$, $\left.J_{\mathrm{d}}=10.7, J_{\mathrm{q}}=7.1 \mathrm{Ha}\right), 3.92\left(1 \mathrm{H}, \mathrm{dq}, J_{\mathrm{d}}=10.7, J_{\mathrm{q}}=7.1 \mathrm{~Hz}\right), 4.14-$ $4.20(2 \mathrm{H}, \mathrm{m}), 4.82(2 \mathrm{H}, \mathrm{s}), 5.80(1 \mathrm{H}, \mathrm{t}, J=11.5 \mathrm{~Hz}), 7.18-7.22$ $(3 \mathrm{H}, \mathrm{m}), 7.35-7.37(2 \mathrm{H}, \mathrm{m}) ;{ }^{13} \mathrm{C}$ NMR $\delta=13.39,13.90,17.94$, $25.84,33.15,51.17,52.74,60.49,61.29,61.38,88.78,114.80$, 127.98, 128.02, 129.84, 135.09, 142.79, 169.87, 169.97. HRMS: $m / z$ 359.1820. Calcd for $\mathrm{C}_{21} \mathrm{H}_{27} \mathrm{O}_{5}: \mathrm{M}-\mathrm{NO}, 359.1858$.

Diethyl 3a,4,5,6,7,8-Hexahydro-3,3-dimethyl-8-phenyl-3H- 
cyclohepta[c]isooxazole-7,7-dicaboxylate 1-Oxide (23): Colorless oil, obtained as a mixture of two diastereomers $(80: 20)$. ${ }^{13} \mathrm{CNMR}$ of the major isomer is only shown. IR (neat) 2979 , $1732,1620,1452,1371,1207,702 \mathrm{~cm}^{-1} ;{ }^{1} \mathrm{HNMR} \delta=1.10$ $(3 \mathrm{H} \times 0.83, \mathrm{t}, J=7.1 \mathrm{~Hz}), 1.14(3 \mathrm{H} \times 0.83, \mathrm{t}, J=7.1 \mathrm{~Hz}), 1.19$ $(3 \mathrm{H} \times 0.17, \mathrm{t}, J=7.1 \mathrm{~Hz}), 1.257(3 \mathrm{H} \times 0.17, \mathrm{~s}), 1.264(3 \mathrm{H} \times 0.17, \mathrm{t}$, $J=7.1 \mathrm{~Hz}), 1.32(3 \mathrm{H} \times 0.83, \mathrm{~s}), 1.37(3 \mathrm{H} \times 0.83, \mathrm{~s}), 1.46(3 \mathrm{H} \times 0.17$, s), $1.65-1.88(4 \mathrm{H}, \mathrm{m}), 2.08-2.17(1 \mathrm{H} \times 0.17, \mathrm{~m}), 2.26-2.30$ $(1 \mathrm{H} \times 0.83, \mathrm{~m}), 2.30-2.37(1 \mathrm{H} \times 0.17, \mathrm{~m}), 2.42-2.47(1 \mathrm{H} \times 0.82$, $\mathrm{m}), 3.22-3.24(1 \mathrm{H} \times 0.83, \mathrm{~m}), 3.29-3.32(1 \mathrm{H} \times 0.17, \mathrm{~m}), 4.00$ $4.18(4 \mathrm{H} \times 0.83+2 \mathrm{H} \times 0.17, \mathrm{~m}), 4.19-4.24(1 \mathrm{H} \times 0.17, \mathrm{~m}), 4.27-$ $4.33(1 \mathrm{H} \times 0.17, \mathrm{~m}), 4.75(1 \mathrm{H} \times 0.83, \mathrm{~s}), 4.78(1 \mathrm{H} \times 0.17, \mathrm{~s}), 7.21-$ $7.34(5 \mathrm{H}, \mathrm{m}) ;{ }^{13} \mathrm{CNMR} \delta=13.84,20.81,22.51,26.56,26.73,32.11$, $48.11,53.15,61.07,61.40,61.77,81.90,120.59,127.75,128.31$, 128.47, 129.44, 129.91, 136.16, 169.46. HRMS: $m / z 403.1997$. Calcd for $\mathrm{C}_{22} \mathrm{H}_{29} \mathrm{NO}_{6}$ : M, 403.1995 .

Typical Procedure for the Substitution of Allylic Hydroxy Group with Silyl Enol Ethers. To a solution of (1-trimethylsiloxyethenyl)benzene (2) $(84.1 \mathrm{mg}, 0.437 \mathrm{mmol})$ in dichloromethane (2 $\mathrm{ml}$ ) was added a solution of trifluoroborane-diethyl ether (1/1) $(0.95$ mol dm${ }^{-3}$ in methanol, $0.08 \mathrm{ml}, 0.076 \mathrm{mmol}$ ), and then a solution of 1,3-dimethyl-2-cyclohexen-1-ol (24) (50.6 mg, $0.401 \mathrm{mmol})$ in dichloromethane $(2 \mathrm{ml})$ slowly at $-78^{\circ} \mathrm{C}$. After stirring for $15 \mathrm{~min}$, the reaction was quenched by adding a saturated aqueous solution of $\mathrm{NaHCO}_{3}$. The mixture was extracted with dichloromethane (10 $\mathrm{ml} \times 4$ ) and dried over anhydrous $\mathrm{Na}_{2} \mathrm{SO}_{4}$. After evaporation of the solvent, the residue was purified by TLC (hexane-ethyl acetate, $20: 1(\mathrm{v} / \mathrm{v}))$ to afford the product, 2-(1,3-dimethyl-2-cyclohexen-1yl)-1-phenyl-1-ethanone (25) (78.4 mg, $0.343 \mathrm{mmol}, 86 \%$ ).

The spectral data of the products are as follows:

2-(1,3-Dimethyl-2-cyclohexen-1-yl)-1-phenyl-1-ethanone (25): Colorless oil; IR (neat) 2925, 1687, 1674, 1597, 1448, 1011, 750, $692 \mathrm{~cm}^{-1} ;{ }^{1} \mathrm{H}$ NMR $\delta=1.09(3 \mathrm{H}, \mathrm{s}), 1.40-1.47(1 \mathrm{H}, \mathrm{m}), 1.57(3 \mathrm{H}$, s), $1.61-1.66(3 \mathrm{H}, \mathrm{m}), 1.84-1.88(2 \mathrm{H}, \mathrm{br}), 2.83(1 \mathrm{H}, \mathrm{d}, J=14.5$ $\mathrm{Hz}), 2.97(1 \mathrm{H}, \mathrm{d}, J=14.5 \mathrm{~Hz}), 5.25(1 \mathrm{H}, \mathrm{br} \mathrm{s}), 7.39-7.42(2 \mathrm{H}$, $\mathrm{m}), 7.48-7.52(1 \mathrm{H}, \mathrm{m}), 7.89-7.91(2 \mathrm{H}, \mathrm{m}) ;{ }^{13} \mathrm{CNMR} \delta=19.44$ $23.80,27.68,29.86,35.12,35.48,49.26,128.24,128.34,129.98$, 132.56, 132.93, 138.68, 200.21. Found: C, $84.32 ; \mathrm{H}, 8.85 \%$. Calcd for $\mathrm{C}_{16} \mathrm{H}_{20} \mathrm{O}: \mathrm{C}, 84.16 ; \mathrm{H}, 8.83 \%$.

1,5-Dimethyl-3-phenyl-2-oxabicyclo[3.3.1]non-3-ene (26): Colorless oil; IR (neat) 3053, 2945, 1641, 1493, 1450, 1327, 1255 , $1176,1052,750,692 \mathrm{~cm}^{-1} ;{ }^{1} \mathrm{HNMR} \delta=1.12(3 \mathrm{H}, \mathrm{s}), 1.27-1.34$ $(1 \mathrm{H}, \mathrm{m}), 1.35-1.40(1 \mathrm{H}, \mathrm{m}), 1.36(3 \mathrm{H}, \mathrm{s}), 1.45-1.58(4 \mathrm{H}, \mathrm{m})$, $1.64-1.74(1 \mathrm{H}, \mathrm{m}), 1.90-1.95(1 \mathrm{H}, \mathrm{br} \mathrm{d}), 4.97(1 \mathrm{H}, \mathrm{d}, J=1.7 \mathrm{~Hz})$, $7.23-7.26(1 \mathrm{H}, \mathrm{m}$ (t-like) $), 7.29-7.32(2 \mathrm{H}, \mathrm{m}$ (t-like) $), 7.59$ $7.61\left(2 \mathrm{H}, \mathrm{m}\right.$ (d-like)); ${ }^{13} \mathrm{C}$ NMR $\delta=20.44,28.75,29.16,31.54$, $38.42,39.55,44.25,75.47,103.82,124.57,127.54,128.04,136.23$, 152.16. HRMS: $m / z$ 228.1502. Calcd for $\mathrm{C}_{16} \mathrm{H}_{20} \mathrm{O}: \mathrm{M}, 228.1514$.

1-(1,3-Dimethyl-2-cyclohexenyl)-3-buten-2-one (27): Colorless oil; IR (neat) 2929, 1689,1612,1454, 1400, $989 \mathrm{~cm}^{-1}$. ${ }^{1} \mathrm{HNMR} \delta=1.03(3 \mathrm{H}, \mathrm{s}), 1.35-1.40(1 \mathrm{H}, \mathrm{m}), 1.53-1.62(3 \mathrm{H}$ m), $1.59(3 \mathrm{H}, \mathrm{s}), 1.81-1.83(2 \mathrm{H}, \mathrm{m}), 2.45(1 \mathrm{H}, \mathrm{d}, J=13.7 \mathrm{~Hz})$, $2.52(1 \mathrm{H}, \mathrm{d}, J=13.7 \mathrm{~Hz}), 5.19(1 \mathrm{H}, \mathrm{s}), 5.69(1 \mathrm{H}, \mathrm{dd}, J=1.1,10.6$ $\mathrm{Hz}), 6.12(1 \mathrm{H}, \mathrm{dd}, J=1.1,17.5 \mathrm{~Hz}), 6.32(1 \mathrm{H}, \mathrm{dd}, J=10.6,17.5$ $\mathrm{Hz}) ;{ }^{13} \mathrm{C}$ NMR $\delta=19.36,23.80,27.72,29.81,35.04,35.23,51.44$, 127.39, 129.66, 133.11, 137.93, 200.40. HRMS: $\mathrm{m} / \mathrm{z}$ 177.1272. Calcd for $\mathrm{C}_{12} \mathrm{H}_{17} \mathrm{O}$ : $\mathrm{M}-\mathrm{H}, 177.1279$.

$\beta$-Nitro ketone 28 was prepared from 27 according to the literature. ${ }^{28)}$

1-(1,3-Dimethyl-2-cyclohexenyl)-4-nitro-2-butanone (28): This compound easily decomposed by elimination of $\mathrm{HNO}_{2}$ to reproduce $\mathbf{2 7}$, so that it was immediately used for the next reaction.
Colorless oil; ${ }^{1} \mathrm{H}$ NMR $\delta=1.06(3 \mathrm{H}, \mathrm{s}), 1.38-1.43(1 \mathrm{H}, \mathrm{m}), 1.49$ $1.55(1 \mathrm{H}, \mathrm{m}), 1.57-1.62(2 \mathrm{H}, \mathrm{m}), 1.63(3 \mathrm{H}, \mathrm{s}), 1.85(2 \mathrm{H}, \mathrm{br}), 2.42$ $(1 \mathrm{H}, \mathrm{d}, J=14.2 \mathrm{~Hz}), 2.46(1 \mathrm{H}, \mathrm{d}, J=14.2 \mathrm{~Hz}), 3.01-3.04(2 \mathrm{H}, \mathrm{m})$, $4.58(2 \mathrm{H}, \mathrm{t}, J=6.0 \mathrm{~Hz}), 5.21(1 \mathrm{H}, \mathrm{br} \mathrm{s}) ;{ }^{13} \mathrm{C} \mathrm{NMR} \delta=19.34,23.87$, $27.82,29.80,34.97,35.04,40.52,54.45,68.99,128.95,134.12$, 205.37 .

Acetal 29 was prepared from 28 according to the literature. ${ }^{29)}$

1-(1,3-Dimethyl-2-cyclohexenyl)-4-nitro-2-butanone Ethylene Acetal (29): $\quad$ Pale yellow oil; IR (neat) 2927, 1556, 1437, $1382,1144,1043 \mathrm{~cm}^{-1} ;{ }^{1} \mathrm{H} N M R \quad \delta=1.00(3 \mathrm{H}, \mathrm{s}), 1.27-1.31(1 \mathrm{H}$, m), $1.57-1.85(7 \mathrm{H}, \mathrm{m}), 1.61(3 \mathrm{H}, \mathrm{s}), 2.45(2 \mathrm{H}, \mathrm{t}, J=6.8 \mathrm{~Hz}), 3.86$ $3.92(4 \mathrm{H}, \mathrm{m}), 4.38(2 \mathrm{H}, \mathrm{t}, J=6.8 \mathrm{~Hz}), 5.19(1 \mathrm{H}, \mathrm{br} \mathrm{s}) ;{ }^{13} \mathrm{CNMR}$ $\delta=19.44,23.88,29.31,29.78,33.93,35.28,35.86,48.33,64.42$, 64.59, 71.06, 110.17, 130.52, 132.53. Found: C, 62.46; H, 8.55; N, 5.36\%. Calcd for $\mathrm{C}_{14} \mathrm{H}_{23} \mathrm{NO}_{4}: \mathrm{C}, 62.43 ; \mathrm{H}, 8.61 ; \mathrm{N}, 5.20 \%$.

Compound $\mathbf{3 0}$ was prepared from 29 according to the procedure for the intramolecular cyclization described before.

$\left(5 \mathrm{a} R^{*}, 8 \mathrm{a} R^{*}, 8 \mathrm{~b} R^{*}\right)-( \pm)-4,4-E$ thylenedioxy-4,5,5a,6,7,8,8a,8boctahydro-5a,8a-dimethyl-3H-naphtho[1,8-cd] isooxazole 2-Oxide (30): Colorless crystals; $\mathrm{mp} 165.0-166.0^{\circ} \mathrm{C}$ (hexane-dichloromethane); IR (KBr) 2958, 1653, 1556, 1440, 1381, 1242, 1103, $785 \mathrm{~cm}^{-1} ;{ }^{1} \mathrm{HNMR} \delta=1.04(3 \mathrm{H}, \mathrm{s}), 1.13-1.17(1 \mathrm{H}, \mathrm{m}), 1.30$ $1.36(1 \mathrm{H}, \mathrm{m}), 1.54(3 \mathrm{H}, \mathrm{s}), 1.54-1.65(3 \mathrm{H}, \mathrm{m}), 1.69-1.75(1 \mathrm{H}$, m), $1.78-1.82(1 \mathrm{H}, \mathrm{m}), 2.09-2.16(1 \mathrm{H}, \mathrm{m}), 2.29(1 \mathrm{H}, \mathrm{dd}, J=4.1$, $16.8 \mathrm{~Hz}), 2.74(1 \mathrm{H}, \mathrm{d}, J=4.1 \mathrm{~Hz}), 2.92(1 \mathrm{H}, \mathrm{d}, J=16.8 \mathrm{~Hz}), 3.81$ $3.85(1 \mathrm{H}, \mathrm{m}), 3.90-3.94(1 \mathrm{H}, \mathrm{m}), 3.96-4.00(2 \mathrm{H}, \mathrm{m}) ;{ }^{13} \mathrm{CNMR}$ $\delta=17.83,25.05,28.75,31.77,33.35,33.76,34.18,47.40,55.77$, $63.74,64.95,82.50,107.60,116.90$. Found: $\mathrm{C}, 62.70 ; \mathrm{H}, 7.69 ; \mathrm{N}$, $5.34 \%$. Calcd for $\mathrm{C}_{14} \mathrm{H}_{21} \mathrm{NO}_{4}: \mathrm{C}, 62.90 ; \mathrm{H}, 7.92 ; \mathrm{N}, 5.24 \%$.

Procedure for the Ozonolysis of 30. Ozone was passed through a solution of $\mathbf{3 0}(17.9 \mathrm{mg}, 0.0700 \mathrm{mmol})$ in methanol $(1 \mathrm{ml})$ at $-78^{\circ} \mathrm{C}$, until the solution was colored blue. The reaction vessel was purged with argon, and then dimethyl sulfide $(0.05 \mathrm{ml})$ was added to the mixture, this was allowed to warm to room temperature. After removal of the solvent, the residue was partitioned with waterether and the aqueous phase was extracted with ether $(10 \mathrm{ml} \times 4)$. The combined extracts were dried over anhydrous $\mathrm{Na}_{2} \mathrm{SO}_{4}$, and then the solvent was evaporated. The residue was purified by flash column chromatography (hexane-ethyl acetate, $2: 1(\mathrm{v} / \mathrm{v})$ ) to afford the product 31 (17.6 mg, $0.0692 \mathrm{mmol}, 99 \%)$.

The spectral data of the product are as follows:

$\left(4 \mathrm{a} R^{*}, 8 R^{*}, 8 \mathrm{a} S^{*}\right)-( \pm)-3,3$-Ethylenedioxy-3,4,4a,5,6,7,8,8aoctahydro-8-hydroxy-4a,8-dimethyl-1 $(2 H)$-naphthalenone (31): Colorless crystals; mp $118.0-120.0{ }^{\circ} \mathrm{C}$ (hexane-dichloromethane); IR (KBr) 3404, 2931, 1684, 1171, $1101 \mathrm{~cm}^{-1}$; ${ }^{1} \mathrm{HNMR}$ $\delta=0.97(3 \mathrm{H}, \mathrm{s}), 1.13-1.17(2 \mathrm{H}, \mathrm{m}), 1.17(3 \mathrm{H}, \mathrm{s}), 1.31-1.34$ $(1 \mathrm{H}, \mathrm{m}), 1.35-1.46(2 \mathrm{H}, \mathrm{m}), 1.57-1.61(2 \mathrm{H}, \mathrm{m}), 1.82-1.91(2 \mathrm{H}$, m), $2.52-2.56(1 \mathrm{H}, \mathrm{m}), 2.79(1 \mathrm{H}$, br d), $3.22(1 \mathrm{H}$, br d), 3.81$3.88(2 \mathrm{H}, \mathrm{m}), 3.93-3.96(2 \mathrm{H}, \mathrm{m}) ;{ }^{13} \mathrm{CNMR} \delta=17.39,30.87$, $31.06,33.98,39.61,40.93,41.06,51.58,63.62,64.67,64.75,70.16$, $110.84,210.71$. Found: C, $65.82 ; \mathrm{H}, 8.72 \%$. Calcd for $\mathrm{C}_{14} \mathrm{H}_{22} \mathrm{O}_{4}$ : C, 66.12; H, 8.72\%.

One of the authors (N. A.) is grateful to Fellowships of the Japan Society for the Promotion of Science for Japanese Junior Scientists. This work was partially supported by a Grant-in-Aid for Scientific Research from the Ministry of Education, Science, Sports and Culture. 


\section{References}

1) B. Giese, "Radicals in Organic Synthesis: Formation of Carbon-Carbon Bonds," Pergamon, Oxford (1986).

2) D. P. Curran, Synthesis, 1988, 417 and 489.

3) P. Renaud and M. A. Fox, J. Org. Chem., 53, 3745 (1988).

4) D. Willemin and A. B. Alloum, Synth. Commun., 22, 3169 (1992).

5) T. Cohen, K. McNamara, M. A. Kuzemko, K. Ramig, J. J. Landi, Jr., and Y. Dong, Tetrahedron, 49, 7931 (1993).

6) A. S. Kende, K. Koch, and C. A. Smith, J. Am. Chem. Soc., 110, 2210 (1988).

7) J. L. Morgat and R. Palloud, C. R. Hebd. Séances Acad. Sci., 260, 5579 (1965).

8) H. J. Schäfer, Angew. Chem., Int. Ed. Engl., 20, 911 (1981); H. Schäfer and A. Al Azrak, Chem. Ber., 105, 2398 (1972).

9) N. Arai and K. Narasaka, Chem. Lett., 1995, 987.

10) W. R. Bowman and S. W. Jackson, Tetrahedron Lett., 30, 1857 (1989).

11) K. Narasaka, K. Iwakura, and T. Okauchi, Chem. Lett., 1991, 423.

12) M. E. Kurz, P. Ngoviwatchai, and T. Tantrarant, J. Org. Chem., 46, 4668 (1981); M. E. Kurz and P. Ngoviwatchai, J. Org. Chem., 46, 4672 (1981).

13) N. Kornblum, H. K. Singh, and W. J. Kelly, J. Org. Chem., 48, 332 (1983).

14) A. S. Kende and K. Koch, Tetrahedron Lett., 27, 6051 (1986).

15) W. R. Bowman, D. S. Brown, C. A. Burns, and D. Crosby, J. Chem. Soc., Perkin Trans. 1, 1993, 2099.

16) M. Montavon, H. Lindlar, R. Marbet, R. Rüegg, G. Ryser, G. Saucy, P. Zeller, and O. Isler, Helv. Chim. Acta, 40, 1250 (1957).

17) D. Seebach and F. Lehr, Angew. Chem., Int. Ed. Engl., 15, 505 (1976).

18) M. V. Proštenik and I. Butula, Angew. Chem., Int. Ed. Engl., 21, 139 (1976).

19) The chemical shift of $\mathbf{4 g}$ showed good agreement with the literature: T. Jeffery-Luong and G. Linstrumelle, Synthesis, 1982, 738.

20) Crystal data for $\mathbf{1 8 b}: \mathrm{C}_{20} \mathrm{H}_{24} \mathrm{BrNO}_{6}, M_{w}=454.32$, colorless prism, monoclinic, $P 2_{1} / a$ (\#14), $a=8.655(3), b=19.595(4), c=$ $12.411(4) \AA, \beta=100.90(2)^{\circ}, V=2067.0(9) \AA^{3}, Z=4, D_{\text {calc }}=1.460$ $\mathrm{g} \mathrm{cm}^{-3}, \mu($ Mo $K \alpha)=20.30 \mathrm{~cm}^{-1}, R=0.075, R_{\mathrm{w}}=0.050,3790$ unique reflections. The structure was solved by direct method (Fan Hai-Fu, "SAPI91," "Structure Analysis Programs with Intelligent Control," Rigaku Corporation, Tokyo, Japan) and expanded using Fourier technique (P. T. Beurskens, G. Admiraal, G. Beurskens, W. P. Bosman, S. Garcia-Granda, R. O. Gould, J. M. M. Smits, and C. Smykalla, "DIRDIF92," "The DIRDIF Program System," Technical Report of the Crystallography Laboratory, University of Nijmegen, The Netherlands). The lists of atomic coordinates, anisotropic parameters for non-hydrogen atoms, and the ORTEP drawing are deposited as Document No. 70039 at the Office of the Editor of Bull. Chem. Soc. Jpn.

21) The calculation was carried out with MacroModel ver.5.5 using MM2* force field: F. Mohamadi, N. G. J. Richards, W. C. Guida, R. Liskamp, C. Caufield, G. Chang, T. Hendrickson, and W. C. Still, J. Comput. Chem., 11, 440 (1990).

22) J. Fossey, D. Lefort, and J. Sorba, "Free Radicals in Organic Chemistry," John Wiley \& Sons, New York (1995).

23) D. N. Hendrickson, Y. S. Sohn, and H. B. Gray, Inorg. Chem., 10, 1559 (1971).

24) M. M. Ray, J. N. Adhya, D. Biswas, and S. N. Podder, Aust. J. Chem., 19, 1737 (1966).

25) T. G. Clarke, N. A. Hampson, J. B. Lee, J. R. Morley, and B. Seanlan, Can. J. Chem., 47, 1649 (1969).

26) H. Sano, M. Okawara, and Y. Ueno, Synthesis, 1984, 933.

27) E. P. Kohler and H. M. Chadwell, Org. Synth., Coll. Vol. 1, 78 (1941).

28) T. Miyakoshi and S. Saito, Nippon Kagaku Kaishi, 1984, 458; T. Miyakoshi, S. Saito, and J. Kumanotani, Chem. Lett., 1981, 1677.

29) G. Rosini, R. Ballini, and P. Sorrenti, Tetrahedron, 39, 4127 (1983). 